Pure and Applied Mathematics Quarterly

Volume 4, Number 3

(Special Issue: In honor of

Fedor Bogomolov, Part 2 of 2$)$

$613-649,2008$

\title{
On the Chow Ring of Certain Algebraic Hyper-Kähler Manifolds
}

\author{
Claire Voisin \\ Pour Fedya Bogomolov, en l'honneur de ses 60 ans
}

\section{INTRODUCTION}

This paper proposes and studies a generalization of a conjecture made by Beauville in [3]. Recall that Beauville and the author proved the following result in [5]:

Theorem 1.1. Let $S$ be an algebraic $K 3$ surface. Then there exists a degree 1 0 -cycle o on $S$ satisfying the property that for any line bundle $L$ on $S$, one has

$$
c_{1}(L)^{2}=\left[c_{1}(L)\right]^{2} o \text { in } C H_{0}(S) .
$$

Furthermore, we have $c_{2}\left(T_{S}\right)=24 o$.

(In this paper, Chern classes will be Chern classes in the Chow ring tensored by $\mathbb{Q}$, and we will denote by $\left[c_{i}\right]$ the corresponding rational cohomology classes.)

This result can be rephrased by saying that any polynomial relation

$$
P\left(\left[c_{1}\left(L_{i}\right)\right]\right)=0 \text { in } H^{*}(S, \mathbb{Q}), L_{i} \in \operatorname{Pic} S,
$$

already holds in $C H(S)$.

In [3], Beauville conjectured that a similar result holds for algebraic hyperKähler varieties:

Received February 22, 2006. 
Conjecture 1.2. (Beauville) Let $Y$ be an algebraic hyper-Kähler variety. Then any polynomial cohomological relation

$$
P\left(\left[c_{1}\left(L_{i}\right)\right]\right)=0 \text { in } H^{*}(Y, \mathbb{Q}), L_{i} \in \operatorname{Pic} Y
$$

already holds at the level of Chow groups :

$$
P\left(c_{1}\left(L_{i}\right)\right)=0 \text { in } C H(Y) .
$$

He proved in [3] this conjecture in the case of the second and third punctual Hilbert scheme of an algebraic $K 3$ surface.

In this paper, we observe that the results of [5] can lead to a more general conjecture concerning the Chow ring of an algebraic hyper-Kähler variety. Namely, the full statement of Theorem 1.1 can be interpreted by saying that any polynomial relation between $\left[c_{2}\left(T_{S}\right)\right],\left[c_{1}\left(L_{i}\right)\right]$ in $H^{*}(S, \mathbb{Q})$, already holds between $c_{2}\left(T_{S}\right), c_{1}\left(L_{i}\right)$ in $C H(S)$. The purpose of this paper is to study the following conjecture:

Conjecture 1.3. Let $Y$ be an algebraic hyper-Kähler variety. Then any polynomial cohomological relation

$$
\left.P\left(\left[c_{1}\left(L_{j}\right)\right],\left[c_{i}\left(T_{Y}\right)\right)\right]\right)=0 \text { in } H^{2 k}(Y, \mathbb{Q}), L_{j} \in \operatorname{Pic} Y
$$

already holds at the level of Chow groups :

$$
P\left(c_{1}\left(L_{j}\right), c_{i}\left(T_{Y}\right)\right)=0 \text { in } C H^{k}(Y) .
$$

We shall prove the following results:

Theorem 1.4. 1) Conjecture 1.3 holds for $Y=S^{[n]}$, for $n \leq 2 b_{2}(S)_{t r}+4$ and any $k$, where $S^{[n]}$ is the Hilbert scheme of length $n$ subschemes of an algebraic $K 3$ surface $S$.

2) Conjecture 1.3 is true for any $k$ when $Y$ is the Fano variety of lines of a cubic fourfold.

In 1), $b_{2}(S)_{t r}=b_{2}(S)-\rho$ is the rank of the transcendental lattice of $S$.

Concerning point 2), recall from [4] that the variety of lines $F$ of a cubic fourfold $X$ is a deformation of $S^{[2]}$, for $S$ an algebraic $K 3$ surface, but that for general $X$, it has Pic $F=\mathbb{Z}$ and thus it is not a Hilbert scheme. Even when 
$\rho(F) \geq 2$ it is not necessarily the case that $F$ is a $S^{[2]}$. In [3], Beauville asked whether his conjecture 1.2 holds true for the variety of lines of a cubic fourfold.

Finally, we also prove the following.

Theorem 1.5. Conjecture 1.3 holds for $Y=S^{[n]}$, and $k=2 n-2,2 n-1,2 n$, for any $S$ as above and any $n$.

The cohomology ring of the Hilbert scheme of a $K 3$ surface has been computed in [14], [16]. For the subring generated by $H^{2}$, on can use the result of Verbitsky [18], [9]. The question of understanding more precisely the Chow ring is rather delicate and we are dealing here only with a small part of it.

We prove in section 1 part 1) of Theorem 1.4 and Theorem 1.5. The proof involves particular cases of the following statement :

Conjecture 1.6. Let $S$ be an algebraic $K 3$ surface. For any integer $m$, let $P \in C H\left(S^{m}\right)$ be a polynomial expression in

$$
p r_{i}^{*} c_{1}\left(L_{s}\right), L_{s} \in P i c S, p r_{j}^{*} o, p r_{k l}^{*} \Delta_{S} .
$$

Then if $[P]=0$, we have $P=0$.

We also prove that Conjecture 1.6 for $S$ and any $m^{\prime} \leq m$ implies Conjecture 1.3 for $Y=S^{[m]}$.

In section 2 we deal with the case of the variety of lines of the cubic fourfold (Theorem 1.4, 2)).

It is a pleasure to dedicate this paper to Fedya Bogomolov, who greatly contributed in the papers [7], [8], [9] to the study of hyper-Kähler manifolds.

\section{Case of the Hilbert scheme of a $K 3$ surface}

Let $S$ be an algebraic $K 3$ surface, and $S^{[n]}$ be the Hilbert scheme of length $n$ subschemes of $S$. For any line bundle $L$ on $S$, there is an induced line bundle, which we still denote by $L$ on $S^{[n]}$, which is the pull-back via the Hilbert-Chow morphism of the line bundle on $S^{(n)}$ corresponding to the $\mathfrak{S}_{n}$-invariant line bundle $L \otimes \ldots \otimes L$ on $S^{n}$. 
There are furthermore two natural vector bundles on $S^{[n]}$, namely $\mathcal{O}_{[n]}$, which is defined as $R^{0} p_{*} \mathcal{O}_{\Sigma_{n}}$, where

$$
\Sigma_{n} \subset S^{[n]} \times S, p=p r_{1}: \Sigma_{n} \rightarrow S^{[n]}
$$

is the incidence scheme, and the tangent bundle $T_{n}$. It is not clear that the Chern classes of $\mathcal{O}_{[n]}$ can be expressed as polynomials in $c_{1}\left(\mathcal{O}_{[n]}\right)$ and the Chern classes of $T_{n}$. The following result may thus be stronger than Theorem 1.4,1):

Theorem 2.1. Let $n \leq 2 b_{2}(S)_{t r}+4$, and let $P \in C H\left(S^{[n]}\right)$ be any polynomial expression in the variables

$$
c_{1}(L), L \in \operatorname{Pic} S \subset \operatorname{Pic} S^{[n]}, c_{i}\left(\mathcal{O}_{[n]}\right), c_{j}\left(T_{n}\right) \in C H\left(S^{[n]}\right) .
$$

Then if $P$ is cohomologous to 0 , we have $P=0$ in $C H\left(S^{[n]}\right)$.

This implies Theorem 1.4 for the $n$-th Hilbert scheme of $K 3$ surface $S$ with $n \leq 2 b_{2}(S)_{t r}+4$, because we have $c_{1}\left(\mathcal{O}_{[n]}\right)=-\delta$, where $2 \delta \equiv E$ is the class of the exceptional divisor of the resolution $S^{[n]} \rightarrow S^{(n)}$, and it is well-known that Pic $S^{[n]}$ is generated by Pic $S$ and $\delta$.

To start the proof of this theorem, we establish first the following Proposition 2.2, which gives particular cases of Conjecture 1.6. Let $o \in C H^{2}(S)$ be the cycle introduced in the introduction. Let $m$ be an integer.

Proposition 2.2. Let $P \in C H\left(S^{m}\right)$ be a polynomial expression in the variables

$$
p r_{i}^{*}\left(\frac{1}{24} c_{2}(T)\right)=p r_{i}^{*} o, p r_{j}^{*} c_{1}\left(L_{s}\right), L_{s} \in \operatorname{Pic} S, p r_{k l}^{*} \Delta_{S}, k \neq l,
$$

where $\Delta_{S} \subset S \times S$ is the diagonal. Assume that one of the following assumptions is satisfied:

(1) $m \leq 2 b_{2}(S)_{t r}+1$.

(2) $P$ is invariant under the action of the symmetric group $\mathfrak{S}_{m-2}$ acting on the $m-2$ first indices.

Then if $P$ is cohomologous to 0 , it is equal to 0 in $C H\left(S^{m}\right)$.

Using the results of [5], this proposition is a consequence of the following lemma: 
Lemma 2.3. The polynomial relations $[P]=0$ in the cohomology ring $H^{*}\left(S^{m}\right)$, satisfying one of the above assumptions on $m, P$, are all generated (as elements of the ring of all polynomial expressions in the variables above) by the following polynomial relations, the list of which will be denoted by $\left(^{*}\right)$ :

(1) $\left[p r_{i}^{*}\left(c_{1}(L)\right) \cdot p r_{i}^{*} o\right]=0, L \in \operatorname{Pic} S,\left[p r_{i}^{*}(o) \cdot p r_{i}^{*}(o)\right]=0$.

(2) $\left[\operatorname{pr}_{i}^{*}\left(c_{1}(L)^{2}-\left[c_{1}(L)\right]^{2} o\right)\right]=0, L \in \operatorname{Pic} S$.

(3) $\left[\operatorname{pr}_{i j}^{*}\left(\Delta_{S} \cdot p_{1}^{*} o-(o, o)\right)\right]=0$, where $p_{1}$ here is the first projection of $S \times S$ to $S$, and $(o, o)=p_{1}^{*} o \cdot p_{2}^{*} o$.

(4) $\left[p_{i j}^{*}\left(\Delta_{S} \cdot p_{1}^{*} c_{1}(L)-c_{1}(L) \times o-o \times c_{1}(L)\right)\right]=0, L \in$ Pic S, where $p_{1}$ here is the first projection of $S \times S$ to $S$, and $c_{1}(L) \times o=p_{1}^{*} c_{1}(L) \cdot p_{2}^{*} O$.

(5) $\left[p r_{i j k}^{*}\left(\Delta_{3}-p_{12}^{*} \Delta_{S} \cdot p_{3}^{*} o-p_{1}^{*} o \cdot p_{23}^{*} \Delta_{S}-p_{13}^{*} \Delta_{S} \cdot p_{2}^{*} O+p_{12}^{*}(o, o)+p_{23}^{*}(o, o)+\right.\right.$ $\left.\left.p_{13}^{*}(o, o)\right)\right]=0$.

(6) $\left[p r_{i j}^{*} \Delta_{S}\right]^{2}=24 p r_{i j}^{*}(o, o)=24 p r_{i}^{*} o \cdot p r_{j}^{*} o$.

In (5), $\Delta_{3}$ is the small diagonal of $S^{3}$ and the $p_{i}, p_{i j}$ are the various projections from $S^{3}$ to $S, S \times S$ respectively. Note that $\Delta_{3}$ can be expressed as $p_{12}^{*} \Delta_{S} \cdot p_{23}^{*} \Delta_{S}$. Furthermore we have

$$
p r_{i j}^{*} \circ p_{1}^{*}=p r_{i}^{*}, p r_{i j k}^{*} \circ p_{12}^{*}=p r_{i j}^{*}, p r_{i j k}^{*} \circ p_{i}^{*}=p r_{i}^{*}
$$

Thus all the relations in $(*)$ are actually polynomial expressions in the variables

$$
\left[p r_{i}^{*} o\right],\left[p r_{j}^{*} c_{1}(L)\right], L \in \operatorname{Pic} S,\left[p r_{k l}^{*} \Delta_{S}\right], k \neq l \text {. }
$$

Assuming this Lemma, we conclude that for $m \leq 2 b_{2}(S)_{t r}+1$, all polynomial relations $[P]=0$ in the variables $p r_{i}^{*} o, p r_{j}^{*} c_{1}(L), L \in P i c S, p r_{k l}^{*} \Delta_{S}, k \neq l$ which hold in $H^{*}\left(S^{m}\right)$ also hold in $C H\left(S^{m}\right)$, because we know from [5] that the relations listed in $\left(^{*}\right)$ hold in $C H\left(S^{m}\right)$. In fact, (apart from the relations (1) and (3) which obviously hold in $C H\left(S^{m}\right)$ ), these relations are pulled-back, via the maps $p_{i}$, resp. $p r_{i j}$, resp. $p r_{i j k}$, from relations in $C H(S)$, resp. $C H\left(S^{2}\right)$, resp. $C H\left(S^{3}\right)$, which are established in [5].

Similarly, for any $m$, the same conclusion holds for polynomial relations invariant under $\mathfrak{S}_{m-2}$.

This concludes the proof of Proposition 2.2. 
Proof of Lemma 2.3. Let $\mathcal{B}$ be a basis of Pic S. It is clear that modulo the relations generated by $(*)$, any polynomial in the variables

$$
\left[p r_{i j}^{*} \Delta_{S}\right],\left[p r_{k}^{*} c_{1}(L)\right], L \in \mathcal{B},\left[p r_{l}^{*} o\right],
$$

can be written as a combination of monomials having the property that an index $i \in\{1, \ldots, n\}$ appears only once. Indeed, these relations express any product with a repeated index as a combination of monomials with no repeated index. Furthermore, if we start from a polynomial which is invariant under the action of $\mathfrak{S}_{m-2}$, as the set of relations $\left(^{*}\right)$ is stable under this action, it is clear that replacing systematically each repeated index by the corresponding combination with no repeated index using $(*)$, we will end with a polynomial expression invariant under the action of $\mathfrak{S}_{m-2}$.

We claim now that if $m \leq 2 b_{2}(S)_{t r}+1$, no non zero combination of monomials with no repeated index vanishes in $H^{*}\left(S^{m}\right)$. Furthermore, for any $m$, no non zero combination of monomials with no repeated index which is invariant under $\mathfrak{S}_{m-2}$ vanishes in $H^{*}\left(S^{m}\right)$.

To prove the claim, consider the transcendental part of $H^{2}(S, \mathbb{Q})$,

$$
H^{2}(S, \mathbb{Q})_{t r}:=N S(S)^{\perp} .
$$

We have the direct sum decomposition

$$
H^{*}(S, \mathbb{Q})=H^{2}(S, \mathbb{Q})_{t r} \oplus H^{*}(S, \mathbb{Q})_{a l g},
$$

where $H^{*}(S, \mathbb{Q})_{\text {alg }}$ is generated by

$$
H^{0}(S, \mathbb{Q}), N S(S)_{\mathbb{Q}}, H^{4}(S, \mathbb{Q}) .
$$

The decomposition (2.2) induces for any $m$ a direct sum decomposition of

$$
H^{*}\left(S^{m}, \mathbb{Q}\right)=H^{*}(S, \mathbb{Q})^{\otimes m}=p r_{1}^{*} H^{*}(S, \mathbb{Q}) \otimes \ldots \otimes p r_{m}^{*} H^{*}(S, \mathbb{Q}) .
$$

Let $\left[\Delta_{S}\right]_{t r}$ be the projection of $\left[\Delta_{S}\right]$ in the direct summand

$$
H^{2}(S, \mathbb{Q})_{t r} \otimes H^{2}(S, \mathbb{Q})_{t r}
$$

of $H^{4}(S \times S, \mathbb{Q})$. Then we have $\left[\Delta_{S}\right]_{t r} \neq 0$ and

$$
\left[\Delta_{S}\right]=\left[\Delta_{S}\right]_{t r}+p_{1}^{*}[o]+p_{2}^{*}[o]+\sum_{i, j \in \mathcal{B}} \alpha_{i j} p_{1}^{*}\left[c_{1}\left(L_{i}\right)\right] \cdot p_{2}^{*}\left[c_{1}\left(L_{j}\right)\right] .
$$

It is then clear that it suffices to prove the claim with $\operatorname{pr}_{i j}^{*}\left[\Delta_{S}\right]$ replaced by $\operatorname{pr}_{i j}^{*}\left[\Delta_{S}\right]_{t r}$ in the set of variables $(2.1)$. 
Let $M$ be a monomial of the form above, and let $I_{M} \subset\{1, \ldots, m\}$ be the set of indices $i$ appearing in $M$ via a diagonal, i.e. for some $l \neq i$, the variable $p r_{i l}^{*}\left[\Delta_{S}\right]_{t r}$ appears in $M$. Then $I_{M}$ is also the unique index set for which the projection of $M$ in

$$
\bigotimes_{i \notin I_{M}} H^{*}(S, \mathbb{Q})_{a l g} \otimes \bigotimes_{i \in I_{M}} H^{2}(S, \mathbb{Q})_{t r}
$$

is non zero. Hence it follows that a relation

$$
\sum_{M} \alpha_{M} M=0
$$

implies for each fixed $I \subset\{1, \ldots, m\}$ (of even cardinality), by projection onto

$$
\bigotimes_{i \notin I} p r_{i}^{*} H^{*}(S, \mathbb{Q})_{a l g} \otimes \bigotimes_{i \in I} p r_{i}^{*} H^{2}(S, \mathbb{Q})_{t r}
$$

a relation of the form

$$
\sum_{M, I_{M}=I} \alpha_{M} M=0
$$

Now note that we can further decompose each term $\bigotimes_{i \notin I} p r_{i}^{*} H^{*}(S, \mathbb{Q})_{\text {alg }}$ using the basis of $H^{*}(S, \mathbb{Q})_{\text {alg }}$ given by $H^{4}(S, \mathbb{Q}), H^{0}(S, \mathbb{Q})$ and the basis $\mathcal{B}$. Then the relation (2.3) decomposes into a sum of relations of the form

$$
\left(\otimes_{j \notin I} p r_{j}^{*} \alpha_{j}\right) \otimes\left(\sum_{I_{M^{\prime}}=I} \alpha_{M^{\prime}}^{\prime} M^{\prime}\right)
$$

for any given $I$ and given set $\left(\alpha_{j}\right)_{j \notin I}$ of chosen elements in the basis

$$
H^{4}(S, \mathbb{Q}), H^{0}(S, \mathbb{Q}), \mathcal{B}
$$

of $H^{*}(S, \mathbb{Q})_{a l g}$. Here each $M^{\prime}$ is a monomial in the $p r_{i j}^{*}\left[\Delta_{S}\right]_{t r}$, and $I_{M^{\prime}}=I$ means that only indices $i, j \in I$ appear in the monomial $M^{\prime}$, and each index $l \in I$ appears exactly once.

Of course, (2.4) is equivalent to the relation

$$
\sum_{I_{M^{\prime}}=I} \alpha_{M^{\prime}}^{\prime} M^{\prime}=0
$$

which has to hold in $H^{2 s}\left(S^{m}, \mathbb{Q}\right)$ or equivalently in $H^{2 s}\left(S^{I}, \mathbb{Q}\right)$. (Here $2 s$ is the cardinality of $I$, and $S^{I}$ is the product of the copies of $S$ indexed by $I$. Thus clearly (2.5) is pulled-back via the projection $S^{m} \rightarrow S^{I}$ from the corresponding relation in $S^{I}$.) 
Now observe that if we started with a polynomial relation invariant under the action of $\mathfrak{S}_{m-2}$, each relation we get in (2.5) is invariant under the symmetric group $\mathfrak{S}_{I}^{\prime}$ permuting the elements of $I$ which are $\leq m-2$.

In conclusion, we are reduced to prove that for each $I \subset\{1, \ldots, m\}$ of cardinality $2 s$, and thus satisfying $2 s \leq m \leq 2 b_{2}(S)_{t r}+1$, there are no relations in $H^{2 s}\left(S_{I}\right)$ between monomials of the form

$$
\prod r_{i j}^{*}\left[\Delta_{S}\right]_{t r}
$$

where each index $i, j \in I$ appears exactly once. Furthermore, for any $m$, there are no $\mathfrak{S}_{I}^{\prime}$-invariant relations in $H^{2 s}\left(S_{I}\right)$ between monomials of the form above.

For the statement concerning $\mathfrak{S}_{I}^{\prime}$-invariant relations, this is obvious, as the symmetric group $\mathfrak{S}_{I}^{\prime}$ acts with at most two distinct orbits on the set of monomials of degree $s$ in the variables $\operatorname{pr}_{i j}^{*}\left[\Delta_{S}\right]_{t r}, i, j \in I$, with no repeated indices, namely, in the case where $m-1, m \in I$, those monomials containing $p_{m-1, m}^{*}\left[\Delta_{S}\right]_{t r}$ and those not containing it. Assume that $m-1, m \in I$, as otherwise the action is transitive and the result is still simpler.

Then the only possible non zero $\mathfrak{S}_{I^{\prime}}^{\prime}$-invariant relation would be of the form:

$$
\beta \sum_{M \in \mathcal{E}} M=\alpha \sum_{M \in \mathcal{F}} M
$$

where $\mathcal{E}$ is the set of monomials not containing $\operatorname{pr}_{m-1, m}^{*}\left[\Delta_{S}\right]_{t r}$, and $\mathcal{F}$ is the set of monomials containing $\operatorname{pr}_{m-1, m}^{*}\left[\Delta_{S}\right]_{t r}$. We identify $I$ with $\{1, \ldots, 2 s\}$ in such a way that $m-1$ is identified with $2 s-1$ and $m$ with $2 s$. Then this gives an identification of $S^{I}$ with $S^{s} \times S^{s}$ and we can consider each $M$ as above as a self-correspondence of $S^{s}$. Let $p_{1}, p_{2}$ be the two projections of $S^{2 s}$ to $S^{s}$. Then each monomial $M$ as above induces a map

$$
\gamma_{M}: H^{2 s, 0}\left(S^{s}\right) \rightarrow H^{2 s, 0}\left(S^{s}\right),
$$

given by

$$
\gamma_{M}(\eta)=p_{1 *}\left(M \cdot p_{2}^{*} \eta\right)
$$

Now we have $\gamma_{M}=0$ when the monomial $M$ has the property that for two indices $i, j \leq s, p r_{i j}^{*}\left[\Delta_{S}\right]_{t r}$ divides $M$, because $\omega^{2}=0$ on $S$. In particular, we have $\gamma_{M}=0$ for $M \in \mathcal{F}$. Thus (2.7) gives

$$
\beta \sum_{M \in \mathcal{E}} \gamma_{M}=0
$$


On the other hand, when the monomial $M$ has the property that for any indices $i \neq j \leq s, p_{i j}^{*}\left[\Delta_{S}\right]_{t r}$ does not divide $M, M$ is of the form

$$
\Pi_{i \leq s} \operatorname{pr}_{i, s+\sigma(i)}^{*}\left[\Delta_{S}\right]_{t r}
$$

for some permutation $\sigma$ of $\{1, \ldots, s\}$. In that case, we have

$$
\gamma_{M}\left(p r_{1}^{*} \omega \cdot \ldots \cdot p r_{s}^{*} \omega\right)=p r_{\sigma(1)}^{*} \omega \cdot \ldots \cdot p r_{\sigma(s)}^{*} \omega=p_{1}^{*} \omega \cdot \ldots \cdot p_{s}^{*} \omega .
$$

Thus we get by (2.9) and (2.10)

$$
\beta \sum_{M \in \mathcal{E}} \gamma_{M}=\beta s ! I d_{H^{2 s, 0}\left(S^{s}\right)}=0,
$$

which implies that $\beta=0$, and that if $\alpha \neq 0$, the relation reduces to $\sum_{M \in \mathcal{F}} M=0$. But elements of $\mathcal{F}$ are of the form

$$
p_{m-1, m}^{*}\left[\Delta_{S}\right]_{t r} \cdot M^{\prime}
$$

where $M^{\prime} \in \mathcal{F}^{\prime}$ are the monomials with no repeated indices in the $\operatorname{pr}_{i j}^{*}\left[\Delta_{S}\right]_{t r}, i, j<$ $m-1, i, j \in I$. The relation $\sum_{M \in \mathcal{F}} M=0$ thus provides $\sum_{M^{\prime} \in \mathcal{F}^{\prime}} M^{\prime}=0$, which has just been proved to be impossible.

In the case where $2 s \leq m \leq 2 b_{2}(S)_{t r}+1$, we have $s \leq b_{2}(S)_{t r}$. The intersection form on $H^{2}(S, \mathbb{C})_{t r}$ is non degenerate. Thus we can choose an orthonormal basis $\alpha_{i}, 1 \leq i \leq b_{2}(S)_{t r}$, of $H^{2}(S, \mathbb{C})_{t r}$. Let

$$
\eta:=p r_{1}^{*} \alpha_{1} \cdot \ldots \cdot p r_{s}^{*} \alpha_{s} .
$$

For each monomial $M$, consider now $\gamma_{M}: H^{2 s}\left(S^{s}, \mathbb{C}\right) \rightarrow H^{2 s}\left(S^{s}, C\right)$, defined as in (2.8). As we have $\left\langle\alpha_{i}, \alpha_{j}\right\rangle=0$ for $i \neq j$, we find that if there are two indices $i, j>s$ such that $p_{i j}^{*}\left[\Delta_{S}\right]_{t r}$ appears in $M$, we have $\gamma_{M}(\eta)=0$, and that the remaining $M$ 's are in one-to-one correspondence with permutations $\sigma$ of $\{1, \ldots, s\}$. Then, as before, we have for such $M$ :

$$
\gamma^{*}(\eta)=p_{\sigma(1)}^{*} \alpha_{1} \cdot \ldots \cdot p_{\sigma(s)}^{*} \alpha_{s}=p_{1}^{*} \alpha_{\sigma^{-1}(1)} \cdot \ldots \cdot p_{s}^{*} \alpha_{\sigma^{-1}(s)} .
$$

As the tensors $p r_{1}^{*} \alpha_{\sigma^{-1}(1)} \cdot \ldots \cdot p r_{s}^{*} \alpha_{\sigma^{-1}(s)}, \sigma \in \mathfrak{S}_{s}$ are linearly independent in $H^{2}(S, \mathbb{C})_{t r}$, we conclude from these two facts that a relation $\sum_{M} \alpha_{M} M=0$ implies $\alpha_{M}=0$ for all those $M$ such that for no indices $i, j>s, p r_{i j}^{*}\left[\Delta_{S}\right]_{t r}$ appears in $M$.

To show that the other coefficients $\alpha_{M}$ must be also 0 , we introduce maps similar to the $\gamma_{M}$, defined by choosing any subset $I_{1}=\left\{i_{1}, \ldots, i_{s}\right\}, i_{1}<\ldots<i_{s}$ of $I$. Denoting by $I_{2}$ the complementary set, we define $\gamma_{M}^{I_{1}}$ as $p_{I_{1} *} \circ\left(M \cdot p_{I_{2}}^{*}\right)$, 
where $p_{I_{1}}$ (resp. $p_{I_{2}}$ ) is the projection from $S_{I} \cong S^{2 s}$ to $S^{s}$ determined by the (ordered) set $I_{1}$ (resp. $I_{2}$ ). For any $M$, there is a choice of $I_{1}$ such that for no indices $i, j \in I_{2}, \operatorname{pr}_{i j}^{*}\left[\Delta_{S}\right]_{t r}$ appears in $M$, and then we conclude as before that $\alpha_{M}$ must also be 0 .

Thus Lemma 2.3 and also Proposition 2.2 are proved.

We come now to the geometry of $S^{[n]}$. Let us introduce the following notation: let

$$
\mu=\left\{\mu_{1}, \ldots, \mu_{m}\right\}, m=m(\mu), \sum_{i}\left|\mu_{i}\right|=n
$$

be a partition of $\{1, \ldots, n\}$. Such a partition determines a partial diagonal

$$
S_{\mu} \cong S^{m} \subset S^{n},
$$

defined by the conditions

$$
x=\left(x_{1}, \ldots, x_{n}\right) \in S_{\mu} \Leftrightarrow x_{i}=x_{j} \text { if } i, j \in \mu_{l}, \text { for some } l .
$$

Consider the quotient map

$$
q_{\mu}: S^{m} \cong S_{\mu} \rightarrow S^{(n)}
$$

and denote by $E_{\mu}$ the following fibered product:

$$
E_{\mu}:=S_{\mu} \times{ }_{S^{(n)}} S^{[n]} \subset S^{m} \times S^{[n]} .
$$

We view $E_{\mu}$ as a correspondence between $S^{m}$ and $S^{[n]}$ and we will denote as usual by $E_{\mu}^{*}: C H\left(S^{[n]}\right) \rightarrow C H\left(S^{m}\right)$ the map

$$
\alpha \mapsto p r_{1 *}\left(p r_{2}^{*}(\alpha) \cdot E_{\mu}\right) .
$$

Let us denote by $\mathfrak{S}_{\mu}$ the subgroup of $\mathfrak{S}_{m}$ permuting only the indices $i, j$ for which the cardinalities of $\mu_{i}, \mu_{j}$ are equal. The group $\mathfrak{S}_{\mu}$ can be seen as the quotient of the global stabilizer of $S_{\mu}$ in $S^{n}$ by its pointwise stabilizer. In this way the action of $\mathfrak{S}_{\mu}$ on $S_{\mu} \cong S^{m}$ is induced by the action of $\mathfrak{S}_{n}$ on $S^{n}$.

We have the following result:

Proposition 2.4. Let $P \in C H\left(S^{[n]}\right)$ be a polynomial expression in $c_{i}\left(\mathcal{O}_{[n]}\right), c_{j}\left(T_{n}\right)$. Then for any $\mu$ as above, $E_{\mu}^{*}(P) \in C H\left(S^{m}\right)$ is a polynomial expression in $p r_{s}^{*} o, p r_{l k}^{*} \Delta_{S}$. Furthermore, $E_{\mu}^{*}(P)$ is invariant under the group $\mathfrak{S}_{\mu}$. 
Note that the last statement is obvious, since $\mathfrak{S}_{\mu}$ leaves invariant the correspondence $E_{\mu} \subset S_{\mu} \times S^{[n]}$.

We postpone the proof of this proposition and conclude the proofs of the theorems.

Proof of Theorem 2.1. From the work of De Cataldo-Migliorini [11], it follows that the map

$$
\left(E_{\mu}^{*}\right)_{\mu \in \operatorname{Part}(\{1, \ldots, n\})}: C H\left(S^{[n]}\right) \rightarrow \Pi_{\mu} C H\left(S^{m(\mu)}\right)
$$

is injective. Let now $P \in C H\left(S^{[n]}\right)$ be a polynomial expression in $c_{1}(L), L \in$ $\operatorname{Pic} S \subset \operatorname{Pic} S^{[n]}, c_{i}\left(\mathcal{O}_{[n]}\right), c_{j}\left(T_{n}\right) \in C H\left(S^{[n]}\right)$. Note first that for $L \in P i c S$, and for each $\mu$, the restriction of $p r_{2}^{*} L$ to $E_{\mu} \subset S_{\mu} \times S^{[n]}$ is a pull-back $p r_{1}^{*} L_{\mu \mid E_{\mu}}$, where $L_{\mu} \in \operatorname{Pic} S_{\mu}=\operatorname{Pic} S^{m}$ is equal to $L^{\otimes\left|\mu_{1}\right|} \otimes \ldots \otimes L^{\otimes\left|\mu_{m}\right|}$. This follows from the fact that $L$ is the pull-back of a line bundle on $S^{(n)}$. Note that $L_{\mu}$ is invariant under $\mathfrak{S}_{\mu}$.

Thus it follows from Proposition 2.4 and the projection formula that for each partition $\mu, E_{\mu}^{*}(P)$ is a polynomial expression in $p r_{i}^{*} c_{1}(L), p r_{k}^{*} o, p r_{l m}^{*} \Delta$ which is invariant under the group $\mathfrak{S}_{\mu}$.

Now, if $P$ is cohomologous to 0 , each $E_{\mu}^{*}(P)$ is cohomologous to 0 . Let us now verify that the assumptions of Proposition 2.2 are satisfied. Recall that we assume $n \leq 2 b_{2}(S)_{t r}+4$. If $m(\mu) \leq 2 b_{2}(S)_{t r}+1$, Proposition 2.2 applies. Otherwise, $m(\mu) \geq 2 b_{2}(S)_{t r}+2$ and, as $n \leq 2 b_{2}(S)_{t r}+4$, it follows that the partition $\mu$ contains at most two sets of cardinality $\geq 2$. Thus the group $\mathfrak{S}_{\mu}$ contains in this case a group conjugate to $\mathfrak{S}_{m(\mu)-2}$. Proposition 2.2 thus applies, and gives $E_{\mu}^{*}(P)=0$ in $C H\left(S_{\mu}\right)$, for all $\mu$.

It follows that $P=0$ by the result of De Cataldo-Migliorini. This concludes the proof of Theorem 2.1.

Proof of Theorem 1.5. Let $P \in C H^{k}\left(S^{[n]}\right)$, with $k \geq 2 n-2$ be a polynomial expression in $c_{1}(L), L \in \operatorname{Pic} S \subset \operatorname{Pic} S^{[n]}, c_{i}\left(\mathcal{O}_{[n]}\right), c_{j}\left(T_{n}\right) \in C H\left(S^{[n]}\right)$, and assume that $[P]=0$. Notice that because $k \geq 2 n-2$, we have $E_{\mu}^{*} P=0$ if the image of $E_{\mu}$ in $S^{[n]}$ has codimension $>2$. This is the case once $m(\mu)<n-2$. On the other hand, if $m(\mu) \geq n-2$, the partition $\mu$ has at most two sets $\mu_{i}$ of cardinality $\geq 2$. Hence for $m(\mu) \geq n-2$, the group $\mathfrak{S}_{\mu}$ contains a group conjugate 
to $\mathfrak{S}_{m(\mu)-2}$. As $\left[E_{\mu}^{*} P\right]=0$, and $E_{\mu}^{*} P$ is a $\mathfrak{S}_{\mu}$-invariant polynomial expression in $p r_{i}^{*} c_{1}(L), p r_{j}^{*} o, p r_{i j}^{*} \Delta_{S}$, Proposition 2.2 thus applies, and gives $E_{\mu}^{*}(P)=0$ in $C H\left(S_{\mu}\right)$ for $m(\mu) \geq n-2$. As we also have $E_{\mu}^{*}(P)=0$ in $C H\left(S_{\mu}\right)$ for $m(\mu)<n-2$, the theorem of De Cataldo-Migliorini shows that $P=0$.

To conclude, let us notice that Proposition 2.4 and the end of the proof of Theorem 2.1 prove the following:

Proposition 2.5. Conjecture 1.6 for $S$, and any $m \leq n$, implies Conjecture 1.3 for $S^{[n]}$.

It remains to prove Proposition 2.4. For the proof, we use the formulas proved in [13], which allow induction on $n$. As in [13], in order to get the result by induction, we will need to introduce a more general induction statement, which is the following: For each integer $l$, we can also consider the correspondence

$$
E_{\mu, l}:=E_{\mu} \times \Delta_{S^{l}}
$$

betweeen $S_{\mu} \times S^{l}$ and $S^{[n]} \times S^{l}$, where $\Delta_{S^{l}}$ is the diagonal of $S^{l}$. On $S^{[n]} \times S^{l}$, we have the natural classes $p r_{0 i}^{*} c_{s}\left(\mathcal{I}_{n}\right)$, where $\mathcal{I}_{n}$ is the ideal sheaf of the universal subscheme $\Sigma_{n} \subset S^{[n]} \times S$, and $p r_{0 i}$ is the projection onto the product of the first factor $S^{[n]}$ and the $i$-th factor of $S^{l}$. We shall denote $p r_{0}$ the projection onto the first factor $S^{[n]}$, and $p r_{i}$ the projection onto the $i$-th factor of $S^{l}$.

The induction statement, which will be proved by induction on $n$, is the following generalization of Proposition 2.4 (which is the $l=0$ case):

Proposition 2.6. Let $P \in C H\left(S^{[n]} \times S^{l}\right)$ be a polynomial expression in

$$
p r_{0}^{*} c_{r}\left(\mathcal{O}_{[n]}\right), p r_{0}^{*} c_{s}\left(T_{n}\right), p r_{0 i}^{*} c_{s}\left(\mathcal{I}_{n}\right), 1 \leq i \leq l .
$$

Then for any $\mu$ as above,

$$
E_{\mu, l}^{*}(P) \in C H\left(S^{m} \times S^{l}\right)=C H\left(S^{m+l}\right)
$$

is a polynomial expression in the $p r_{j}^{*} o, p r_{i k}^{*} \Delta, i, j, k \leq m+l$.

Proof. Consider the smooth variety $S^{[n, n-1]}$ parameterizing pairs $\left(z, z^{\prime}\right)$ of subschemes of $S$, of length $n$ and $n-1$ respectively, such that $z^{\prime} \subset z$.

$S^{[n, n-1]}$ admits a natural map $\rho$ to $S$, which to $\left(z, z^{\prime}\right)$ associates the residual point of $z^{\prime}$ in $z$. Together with the two natural projections $\psi$ to $S^{[n]}$ and $\phi$ to 
$S^{[n-1]}$ respectively, this gives two maps:

$$
\psi: S^{[n, n-1]} \rightarrow S^{[n]}, \sigma=(\phi, \rho): S^{[n, n-1]} \rightarrow S^{[n-1]} \times S .
$$

$\sigma$ is birational; in fact it is the blow-up of $S^{[n-1]} \times S$ along the incidence subscheme $\Sigma_{n-1} \subset S^{[n-1]} \times S$. We shall denote by $\mathcal{L}$ the line bundle $\mathcal{O}(-E)$ on $S^{[n, n-1]}$, where $E$ is the exceptional divisor of $\sigma$. Thus we have

$$
\operatorname{Im}\left(\sigma^{*} \mathcal{I}_{n-1} \rightarrow \mathcal{O}_{S^{[n, n-1]}}\right)=\mathcal{O}(-E)=\mathcal{L} .
$$

The map $\psi$ has degree $n$, and $(\psi, \rho)$ is a birational map from $S^{[n, n-1]}$ to the incidence subscheme $\Sigma_{n} \subset S^{[n]} \times S$.

Let now $\mu=\left\{\mu_{1}, \ldots, \mu_{m}\right\}$ be a partition of $\{1, \ldots, n\}$, and $S_{\mu} \cong S^{m} \subset S^{n}$ be as above. Consider the fibered product

$$
S_{\mu} \times_{S^{(n)}} S^{[n, n-1]},
$$

which is also equal to

$$
E_{\mu} \times_{S^{[n]}} S^{[n, n-1]} .
$$

It obviously has exactly $m$ components dominating $S_{\mu}$, according to the choice of the residual point. Let us choose one component, say the one where over the generic point $\left(x_{1}, \ldots, x_{n}\right) \in S_{\mu}$, the residual point is $x_{n}$. Let $\mu^{\prime}$ be the partition of $\{1, \ldots, n-1\}$ deduced from $\mu$ by putting

$$
\mu_{i}^{\prime}=\mu_{i}, \text { if } n \notin \mu_{i}, \mu_{i}^{\prime}=\mu_{i} \backslash\{n\} \text {, if } n \in \mu_{i} .
$$

Let us denote by $E_{\mu, \mu^{\prime}} \subset S_{\mu} \times S^{[n, n-1]}$ the underlying reduced variety of the component defined above, and note that via the projection $\pi$ from $S_{\mu}$ to $S_{\mu^{\prime}}$ (forgetting the $n$-th factor), and the map $\sigma$, we get a natural map

$$
\chi_{\mu^{\prime}}=(\pi, \sigma)_{\mid E_{\mu, \mu^{\prime}}}: E_{\mu, \mu^{\prime}} \rightarrow E_{\mu^{\prime}} \times S .
$$

On the other hand, we have the natural map

$$
\chi_{\mu}:=\left(I d_{S_{\mu}}, \psi\right)_{\mid E_{\mu, \mu^{\prime}}}: E_{\mu, \mu^{\prime}} \rightarrow E_{\mu} .
$$

Now, observe that the following diagram is commutative:

$$
\begin{array}{cccc}
E_{\mu} \stackrel{\chi_{\mu}}{\longleftarrow} E_{\mu, \mu^{\prime}} \stackrel{\chi_{\mu^{\prime}}}{\longrightarrow} & E_{\mu^{\prime}} \times S \\
p_{\mu} \downarrow & & & \downarrow\left(p_{\mu^{\prime}}, I d\right), \\
S_{\mu} & \stackrel{\pi^{\prime}}{\rightarrow} & & S_{\mu^{\prime}} \times S
\end{array}
$$

where $p_{\mu}$ is the restriction to $E_{\mu} \subset S_{\mu} \times S^{[n]}$ of the first projection, and similarly for $p_{\mu^{\prime}}$, and where $\pi^{\prime}: S_{\mu} \rightarrow S_{\mu^{\prime}} \times S$ is given by $\left(\pi, p r_{n \mid S_{\mu}}\right)$. Note also that 
both $\chi_{\mu}$ and $\chi_{\mu^{\prime}}$ are generically finite of degree 1 . Thus we have the following equalities :

$$
\begin{gathered}
E_{\mu, \mu^{\prime}}^{*} \circ \psi^{*}=E_{\mu}^{*}: C H\left(S^{[n]}\right) \rightarrow C H\left(S_{\mu}\right), \\
\pi_{*}^{\prime} \circ E_{\mu, \mu^{\prime}}^{*} \circ \sigma^{*}=\left(E_{\mu^{\prime}} \times \Delta_{S}\right)^{*}: C H\left(S^{[n-1]} \times S\right) \rightarrow C H\left(S_{\mu^{\prime}} \times S\right) .
\end{gathered}
$$

Similarly, for any integer $l$, we can consider the induced correspondence

$$
E_{\mu, \mu^{\prime}, l}:=E_{\mu, \mu^{\prime}} \times \Delta_{S^{l}}
$$

between $S_{\mu} \times S^{l}$ and $S^{[n, n-1]} \times S^{l}$. Then we have the formulas

(2.11) $E_{\mu, l}^{*}=E_{\mu, \mu^{\prime}, l}^{*} \circ\left(\psi, I d_{l}\right)^{*}: C H\left(S^{[n]} \times S^{l}\right) \rightarrow C H\left(S_{\mu} \times S^{l}\right)$,

$(2.12) E_{\mu^{\prime}, l+1}^{*}=\pi_{l *}^{\prime} \circ E_{\mu, \mu^{\prime}, l}^{*} \circ\left(\sigma, I d_{l}\right)^{*}: C H\left(S^{[n-1]} \times S^{l+1}\right) \rightarrow C H\left(S_{\mu^{\prime}} \times S^{l+1}\right)$.

Here, $I d_{l}$ denotes the identity of $S^{l}$, and $\pi_{l}^{\prime}$ is defined by

$$
\pi_{l}^{\prime}=\left(\pi^{\prime}, I d_{l}\right): S_{\mu} \times S^{l} \rightarrow S_{\mu^{\prime}} \times S^{l+1} .
$$

Furthermore, for any $\gamma \in C H\left(S^{[n, n-1]} \times S^{l}\right)$, one has

$$
\pi_{l *}^{\prime} \circ E_{\mu, \mu^{\prime}, l}^{*}(\gamma)=E_{\mu^{\prime}, l+1}^{*}\left(\left(\sigma, I d_{l}\right)_{*} \gamma\right) .
$$

Indeed, this follows from the fact that the correspondences $E_{\mu, \mu^{\prime}} \subset S_{\mu} \times S^{[n, n-1]}$ and $E_{\mu^{\prime}} \times \Delta_{S} \subset S_{\mu^{\prime}} \times S \times S^{[n-1]} \times S$ satisfy the relation:

$$
\left(\pi^{\prime}, I d_{S^{[n, n-1]}}\right)_{*}\left(E_{\mu, \mu^{\prime}}\right)=\left(I d_{S_{\mu^{\prime}}}, \sigma, I d_{S}\right)^{*}\left(E_{\mu^{\prime}} \times \Delta_{S}\right)
$$

in $C H\left(S_{\mu^{\prime}} \times S \times S^{[n, n-1]}\right)$ and similarly with $l>0$. From (2.14), we deduce that for $\gamma \in C H\left(S^{[n, n-1]}\right)$, one has

$$
\begin{gathered}
\pi_{*}^{\prime} \circ E_{\mu, \mu^{\prime}}^{*}(\gamma)=\left(p_{S_{\mu^{\prime}} \times S}\right)_{*}\left(\left(\pi^{\prime}, I d_{S^{[n, n-1]}}\right)_{*}\left(E_{\mu, \mu^{\prime}}\right) \cdot p_{S^{[n, n-1]}}^{*} \gamma\right) \\
=\left(p_{S_{\mu^{\prime}} \times S}\right)_{*}\left(\left(I d_{S_{\mu^{\prime}}}, \sigma, I d_{S}\right)^{*}\left(E_{\mu^{\prime}} \times \Delta_{S}\right) \cdot p_{S^{[n, n-1]}}^{*} \gamma\right) \\
=\left(p_{S_{\mu^{\prime}} \times S}\right)_{*} \circ\left(I d_{S_{\mu^{\prime}}}, \sigma, I d_{S}\right)_{*}\left(\left(I d_{S_{\mu^{\prime}}}, \sigma, I d_{S}\right)^{*}\left(E_{\mu^{\prime}} \times \Delta_{S}\right) \cdot p_{S^{[n, n-1]}}^{*} \gamma\right) \\
=\left(p_{S_{\mu^{\prime}} \times S}\right)_{*}\left(\left(E_{\mu^{\prime}} \times \Delta_{S}\right) \cdot\left(I d_{S_{\mu^{\prime}}}, \sigma, I d_{S}\right)_{*}\left(p_{S^{[n, n-1]}}^{*} \gamma\right)\right) \\
=\left(p_{S_{\mu^{\prime}} \times S}\right)_{*}\left(\left(E_{\mu^{\prime}} \times \Delta_{S}\right) \cdot p_{S^{[n-1]} \times S}^{*}\left(\sigma_{*} \gamma\right)\right) \\
=E_{\mu^{\prime}, 1}^{*}\left(\sigma_{*} \gamma\right),
\end{gathered}
$$

which proves $(2.13)$ for $l=0$. One argues similarly for $l>0$.

From (2.13), using the projection formula, one deduces that for any

$$
\alpha \in C H\left(S^{[n, n-1]} \times S^{l}\right), \beta \in C H\left(S^{[n-1]} \times S^{l+1}\right),
$$


one has :

$$
\pi_{l *}^{\prime} \circ E_{\mu, \mu^{\prime}, l}^{*}\left(\alpha \cdot\left(\sigma, I d_{l}\right)^{*} \beta\right)=E_{\mu^{\prime}, l+1}^{*}\left(\left(\sigma, I d_{l}\right)_{*} \alpha \cdot \beta\right) .
$$

The key point is now the following formulas proved by Ellingsrud, Göttsche, Lehn in [13]: here we work on the $K_{0}$ groups (the varieties considered are smooth and projective). The morphism $\phi^{!}: K_{0}(Y) \rightarrow K_{0}(X)$ for a morphism $\phi: X \rightarrow Y$ between smooth varieties is induced by the morphism $\phi^{*}$ on vector bundles. The morphism $M \mapsto M^{\vee}$ is induced by the morphism $E \mapsto E^{*}$ on vector bundles, and the product $\cdot$ is induced by the tensor product between vector bundles. Then we have (here we use for simplicity the fact that $K_{S}$ is trivial) :

Theorem 2.7. ([13], Lemma 2.1 and Proposition 2.3) We have in $K_{0}\left(S^{[n, n-1]}\right)$ :

$$
\psi^{!} T_{n}=\phi^{!} T_{n-1}+\mathcal{L} \cdot \sigma^{!} \mathcal{I}_{n-1}^{\vee}-\rho^{!}\left(1-T_{S}\right) .
$$

$$
\psi^{!} \mathcal{O}_{[n]}=\phi^{!} \mathcal{O}_{[n-1]}+\mathcal{L} .
$$

Furthermore, we have in $K_{0}\left(S^{[n, n-1]} \times S\right)$ :

$$
\left(\psi, I d_{S}\right)^{!} \mathcal{I}_{n}=\left(\phi, I d_{S}\right)^{!} \mathcal{I}_{n-1}-\operatorname{pr}_{0}^{!}(\mathcal{L}) \otimes\left(\rho, I d_{S}\right)^{!} \mathcal{O}_{\Delta_{S}} .
$$

Another very important property is

Lemma 2.8. ([13], Lemma 1.1) In $C H\left(S^{[n-1]} \times S\right)$, we have the relation

$$
\sigma_{*}\left(c_{1}(\mathcal{L})^{i}\right)=(-1)^{i} c_{i}\left(-\mathcal{I}_{n-1}\right) .
$$

Theorem 2.7 can be translated into statements concerning the Chern classes of the considered sheaves (or elements of the $K_{0}$ groups). Namely we conclude from (2.16) that the Chern classes $c_{i}\left(T_{n}\right)$ satisfy the property that $\psi^{*} c_{i}\left(T_{n}\right)$ can be expressed as polynomials in

$$
\phi^{*} c_{j}\left(T_{n-1}\right), c_{1}(\mathcal{L}), \sigma^{*} c_{s}\left(\mathcal{I}_{n-1}\right), \rho^{*} c_{2}\left(T_{S}\right)=24 \rho^{*} o .
$$

Similarly, we get from (2.17) that the Chern classes $c_{i}\left(\mathcal{O}_{[n]}\right)$ satisfy the property that $\psi^{*} c_{i}\left(\mathcal{O}_{[n]}\right)$ can be expressed as polynomials in

$$
\phi^{*} c_{j}\left(\mathcal{O}_{[n-1]}\right), c_{1}(\mathcal{L}) .
$$

Finally, from (2.18) we conclude that the Chern classes of $\mathcal{I}_{n}$ satisfy the property that $\left(\psi, I d_{S}\right)^{*} c_{i}\left(\mathcal{I}_{n}\right) \in C H\left(S^{[n, n-1]} \times S\right)$ can be expressed as polynomials in

$$
\left(\phi, I d_{S}\right)^{*} c_{j}\left(\mathcal{I}_{n-1}\right), p r_{0}^{*} c_{1}(\mathcal{L}),\left(\rho, I d_{S}\right)^{*} c_{s}\left(\mathcal{O}_{\Delta_{S}}\right) .
$$


Note that because $K_{S}$ is trivial, the Chern classes of $\mathcal{O}_{\Delta_{S}}$ reduce to

$$
c_{2}\left(\mathcal{O}_{\Delta_{S}}\right)=-\Delta_{S} \in C H^{2}(S \times S)
$$

and $c_{4}\left(\mathcal{O}_{\Delta_{S}}\right)$, which is proportional to $(o, o)$ as $c_{2}\left(T_{S}\right)$ is proportional to $o$.

Let now $P \in C H\left(S^{[n]} \times S^{l}\right)$ be a polynomial expression in

$$
p r_{0}^{*} c_{r}\left(\mathcal{O}_{[n]}\right), p r_{0}^{*} c_{s}\left(T_{n}\right), p r_{0 i}^{*} c_{t}\left(\mathcal{I}_{n}\right), 1 \leq i \leq l
$$

as in Proposition 2.6. Applying (2.11), we get

$$
E_{\mu, l}^{*}(P)=E_{\mu, \mu^{\prime}, l}^{*} \circ\left(\psi, I d_{l}\right)^{*}(P) .
$$

As just explained above, $\left(\psi, I d_{l}\right)^{*}(P) \in C H\left(S^{[n, n-1]} \times S^{l}\right)$ can be expressed as a polynomial in

$$
\begin{gathered}
\left(\phi, p r_{i}\right)^{*} c_{t}\left(\mathcal{I}_{n-1}\right), p r_{0}^{*} c_{1}(\mathcal{L}),\left(\phi \circ p r_{0}\right)^{*} c_{r}\left(\mathcal{O}_{[n-1]}\right),\left(\phi \circ p r_{0}\right)^{*} c_{s}\left(T_{n-1}\right), \\
\left(p r_{1, i} \circ\left(\sigma, I d_{l}\right)\right)^{*} \Delta_{S},\left(p r_{i} \circ\left(\sigma, I d_{l}\right)\right)^{*} o, 1 \leq i \leq l+1 .
\end{gathered}
$$

Observing that

$$
\phi \circ p r_{0}: S^{[n, n-1]} \times S^{l} \rightarrow S^{[n-1]}
$$

is equal to $\operatorname{pr}_{0} \circ\left(\sigma, I d_{l}\right)$, the variables above can all be expressed as pull-back via $\left(\sigma, I d_{l}\right)$ of the following variables in $C H\left(S^{[n-1]} \times S^{l+1}\right)$ :

$$
\begin{array}{r}
p r_{1, i}^{*} \Delta_{S}, p r_{i}^{*} o, 1 \leq i \leq l+1, \\
p r_{0 i}^{*} c_{t}\left(\mathcal{I}_{n-1}\right), p r_{0}^{*} c_{r}\left(\mathcal{O}_{[n-1]}\right), p r_{0}^{*} c_{s}\left(T_{n-1}\right),
\end{array}
$$

except for $p r_{0}^{*} c_{1}(\mathcal{L})$. Thus we have in $C H\left(S^{[n, n-1]} \times S^{l}\right)$ :

$$
\left(\psi, I d_{l}\right)^{*}(P)=\sum_{i} p r_{0}^{*} c_{1}(\mathcal{L})^{i}\left(\sigma, I d_{l}\right)^{*} Q_{i},
$$

where $Q_{i} \in C H\left(S^{[n-1]} \times S^{l+1}\right)$ is a polynomial expression in the variables $(2.20)$.

From (2.21) and (2.19), applying (2.15), we deduce that

$$
\pi_{l *}^{\prime}\left(E_{\mu, l}^{*}(P)=\pi_{l *}^{\prime}\left(E_{\mu, \mu^{\prime}, l}^{*} \circ\left(\psi, I d_{l}\right)^{*}(P)\right)=\right.
$$

$$
\pi_{l *}^{\prime}\left(E_{\mu, \mu^{\prime}, l}\right)^{*}\left(\sum_{i} \operatorname{pr}_{0}^{*} c_{1}(\mathcal{L})^{i}\left(\sigma, I d_{l}\right)^{*} Q_{i}\right)=E_{\mu^{\prime}, l+1}^{*}\left(\sum_{i} Q_{i} \cdot\left(\sigma, I d_{l}\right)_{*}\left(\operatorname{pr}_{0}^{*} c_{1}(\mathcal{L})^{i}\right)\right) .
$$

Using Lemma 2.8, we find that $\left.\left(\sigma, I d_{l}\right)_{*}\left(p r_{0}^{*} c_{1}(\mathcal{L})^{i}\right)\right)$ is a polynomial expression in the $p r_{0 j}^{*} c_{s}\left(\mathcal{I}_{n-1}\right)$, and thus

$$
\sum_{i} Q_{i} \cdot\left(\sigma, I d_{l}\right)_{*}\left(p r_{0}^{*} c_{1}(\mathcal{L})^{i}\right)
$$


is a polynomial expression in the variables (2.20). Applying induction on $n$ and the projection formula to the right hand side, we conclude that $\pi_{l *}^{\prime}\left(E_{\mu, l}^{*}(P)\right)$ is a polynomial expression in the variables

$$
p r_{j}^{*} o, p r_{i k}^{*} \Delta_{S}, i, j, k, \leq l+m .
$$

There are finally two cases to consider here, according to whether $|\mu(n)|=1$ or $|\mu(n)| \geq 2$, where $\mu(n)$ is the element of the partition $\mu$ to which $n$ belongs (so $|\mu(n)|$ is the multiplicity of $n$ in the diagonal $S_{\mu}$ ). In the first case, we have

$$
\pi^{\prime}: S_{\mu} \cong S_{\mu^{\prime}} \times S
$$

while in the second case, we have $\pi: S_{\mu} \cong S_{\mu^{\prime}}$ and $\pi^{\prime}$ is the embedding of $S_{\mu} \cong S^{m}$ in $S_{\mu^{\prime}} \times S \cong S^{m+1}$ which is given by the diagonal on the last factor. In the first case, $\pi^{\prime}$ being an isomorphism, we proved that $E_{\mu, l}^{*}(P)$ is a polynomial in the variables $p r_{i j}^{*} \Delta_{S}, p r_{k}^{*} o$. In the second case, we get that $p r_{\mu^{\prime}} \circ \pi^{\prime}$ is an isomorphism from $S_{\mu}$ to $S_{\mu^{\prime}}$, and applying $\left(p r_{\mu^{\prime}}, I d_{l}\right)$ to both sides of (2.22), we get the same conclusion.

This proves Proposition 2.6, and thus also Proposition 2.4.

Remark 2.9. It is presumably the case that Proposition 2.4 could be obtained as a consequence of the Bridgeland-King-Reid-Haiman equivalence of categories between the derived category of $S^{[n]}$ and the derived category of $\mathfrak{S}_{n}$-equivariant coherent sheaves on $S^{n}$ (see [10], [15]), combined with results on equivariant $K$ theory of Vistoli [20], and Riemann-Roch type theorems by Toen [19].

However, the explicit computation of the equivariant complex associated to a given sheaf on $S^{[n]}$ is rather complicated. It is done in [17] for $\mathcal{O}_{[n]}$, but not for $T_{n}$, and the computation is more difficult than the method of [13], that we have been using here.

\section{Case of the variety of lines of a Cubic Fourfold}

We shall use the following notations: the cubic fourfold will be denoted by $X$ and its Fano variety of lines by $F . F$ is contained in the Grassmannian $G:=G(2,6)$ of lines in $\mathbb{P}^{5}$, and we shall denote by

$$
l \in C H^{1}(F, \mathbb{Z}), c \in C H^{2}(F, \mathbb{Z})
$$


the Chern classes of the rank 2 quotient bundle $\mathcal{E}$ induced on $F$. Thus if

$$
\begin{aligned}
& P \stackrel{q}{\rightarrow} X \\
& p \downarrow \\
& F
\end{aligned}
$$

is the incidence diagram, $P$ is a $\mathbb{P}^{1}$-bundle over $F$, and $\mathcal{E}=R^{0} p_{*} q^{*}\left(\mathcal{O}_{X}(1)\right)$.

We shall denote by $H \in C H^{1}(X)$ the class $c_{1}\left(\mathcal{O}_{X}(1)\right)$ and by $h$ its pull-back to $P, h=q^{*} H$.

Let $I \subset F \times F$ be the incidence subvariety, which is the codimension 2 subset of $F \times F$ defined as

$$
I=(p, p)(q, q)^{-1}\left(\Delta_{X}\right),
$$

where $\Delta_{X}$ is the diagonal of $X$. Thus $I$ is the set of pairs $\left(\delta, \delta^{\prime}\right)$ of intersecting lines. We shall denote by the same letter $I$ the class of $I$ in $C H^{2}(F \times F)$.

We start the proof with a few remarks concerning the Chern classes of $F$. As it is known that $F$ is symplectic holomorphic, one has $T_{F} \cong \Omega_{F}$, and thus only the even Chern classes of $F$ can be non zero. We shall denote them by $c_{2}, c_{4}$. It is immediate to compute that $c_{2}$ and $c_{4}$ can be written as polynomials in $c$ and $l$. Indeed $F \subset G$ is defined as the zero set of a section of the vector bundle $S^{3} \mathcal{E}_{G}$ on $G$, and thus the normal bundle of $F$ in $G$ is isomorphic to $S^{3} \mathcal{E}$. The normal bundle exact sequence then shows that the Chern classes of $F$ are polynomials in $l, c$ and in the Chern classes of $G$ restricted to $F$. But the later are also polynomials in $c$ and $l$, as are the restrictions of all cycles on the Grassmannian.

Thus, in this case, Theorem 1.4, 2) is equivalent to the following:

Theorem 3.1. Any polynomial expression in $D \in C H^{1}(F)$ and $c \in C H^{2}(F)$ which vanishes in cohomology, vanishes in $C H(F)$.

We observe first that there is no cohomological relation in degree 4 of the form above. Indeed, as $F$ is a deformation of a $S^{[2]}$, one knows that

$$
H^{4}(F, \mathbb{Q}) \cong S^{2} H^{2}(F, \mathbb{Q}) .
$$

Thus there is only one cohomological relation of the form

$$
\left[c_{2}(F)\right]=P,
$$


where $P \in S^{2} H^{2}(F, \mathbb{Q})$. But this $P$ is non degenerate because its kernel is a sub-Hodge structure of $H^{2}(F, \mathbb{Q})^{*}$, which must be trivial because it is stable under deformation of $F$, and in particular under a deformation for which $N S(F)$ becomes trivial. Thus there cannot be any relation of the form

$$
\left[c_{2}(F)\right]=Q
$$

where $Q \in S^{2}(N S(F))$, because $N S(F)$ never generates $H^{2}(F, \mathbb{Q})$.

Thus we only have to study relations in $H^{6}$ and $H^{8}$. We first deal with the relations between $l$ and $c$ in degree 8 . There are obviously two such relations, as $l^{4}, c^{2}, l^{2} c$ are all proportional in $H^{8}(F, \mathbb{Q})$. Let us prove:

Lemma 3.2. There exists a 0 -cycle $o \in C H^{4}(F)$, which is of degree 1 , and such that

$$
l^{4}, c^{2}, l^{2} c
$$

are multiples of o in $C H^{4}(F)$.

Proof. We observe first that for generic $X$, there is one surface $\Sigma$ of class $c$ which is a singular rational surface (namely, its desingularization is rational). Indeed, surfaces in the class $c$ are surfaces of lines of hyperplane sections of $X$. When an hyperplane section $Y$ acquires a node $x$, its surface of lines becomes birationally equivalent to a symmetric product $S^{2} E_{x}$, where $E_{x}$ is the curve of lines in $Y$ (or $X$ ) passing through $x$ (see [12]). This curve of lines has genus 4, and imposing four "independent" supplementary nodes to $Y$ creates four nodes on the curve $E_{x}$, which remains irreducible, so that the normalization of $E_{x}$ becomes rational. In that case, the desingularization of the surface of lines of $Y$ is rational. Now, for generic $X$ it is easy to see that there exists such an hyperplane section $Y$ with five independent nodes (which means that the associated vanishing cycles are independent).

Of course, all points of $\Sigma$ are rationally equivalent in $F$. For some particular $X$, it might be that the surface $\Sigma$ degenerates to a non rational surface, but it still will remain true that all the points of the degenerate surface $\Sigma$ are rationally equivalent in $F$.

We shall denote by $o \in C H^{4}(F)$ this degree 10 -cycle. As $c^{2}$ is supported on $\Sigma, c^{2}$ is a multiple of $o$ in $C H^{4}(F)$. Similarly $c \cdot l^{2}$ is supported on $\Sigma$, hence it has to be a multiple of $o$ in $C H^{4}(F)$. 
Next, with the same notations as above, we note that the curve $E_{x}$ is contained in $\Sigma$. Thus we have a relation in $C H^{4}(X)$ :

$$
l \cdot E_{x}=\mu o,
$$

for some coefficient $\mu$ equal to the degree of $l \cdot E_{x}$. The class of $E_{x}$ is computed as follows: As $C H_{0}(X)=\mathbb{Z}$, this class does not depend on $x$, and in fact we have:

$$
3 E_{x}=p_{*} h^{4},
$$

because $3 x$ is rationally equivalent to $H^{4}$ in $X$. Now we have the relation defining Chern classes:

$$
\left(p^{*} l-h\right) h=p^{*} c
$$

in $C H^{2}(P)$, which gives

$$
\begin{aligned}
& h^{2}=p^{*} l \cdot h-p^{*} c, h^{3}=p^{*} l \cdot\left(p^{*} l \cdot h-p^{*} c\right)-h \cdot p^{*} c=p^{*}\left(l^{2}-c\right) \cdot h-p^{*}(l \cdot c), \\
& h^{4}=p^{*}\left(l^{2}-c\right) \cdot\left(p^{*} l \cdot h-p^{*} c\right)-p^{*}(l \cdot c) \cdot h=p^{*}\left(l^{3}-2 l c\right) \cdot h-p^{*}\left(\left(l^{2}-c\right) c\right) .
\end{aligned}
$$

Thus we have

$$
3 E_{x}=l^{3}-2 l c \text { in } C H^{3}(F) .
$$

Equation (3.25) thus gives a relation

$$
3 l\left(l^{3}-2 l c\right)=\mu o,
$$

and thus $l^{4}$ is also a multiple of $o$.

We now introduce a relation in the Chow ring of $F \times F$ which generalizes the results obtained in [22] (which concerned in particular the Chow ring of the surface of lines of a cubic threefold). This relation will be essential to understand the group $C H_{1}(F)$.

Proposition 3.3. There is a quadratic relation in $C H^{4}(F \times F)$

$$
I^{2}=\alpha \Delta_{F}+\Gamma \cdot I+\Gamma^{\prime},
$$

where $\alpha \neq 0$, and $\Gamma$ is a codimension 2 cycle of $F \times F$ which is a degree 2 polynomial in

$$
l_{1}:=p_{1}^{*} l, l_{2}=p_{2}^{*} l,
$$

and $\Gamma^{\prime}$ is a codimension 4 cycle which is a degree 2 weighted polynomial in $l_{1}, l_{2}, p_{1}^{*} c, p_{2}^{*} c$. 
Proof. We first prove the existence of a relation of the above form, and we will show later on that the coefficient $\alpha$ is not 0 .

To get such a relation, it suffices to show the existence of a relation

$$
I_{0}^{2}=\Gamma \cdot I_{0}+\Gamma^{\prime} \text { in } C H^{4}\left(F \times F \backslash \Delta_{F}\right),
$$

where $\Gamma, \Gamma^{\prime}$ are as above and $I_{0}$ is the restriction of $I$ to $F \times F \backslash \Delta_{F}$.

Note that $I$ is the image in $F \times F$ via the map $(p, p)$ of

$$
\widetilde{I}:=(q, q)^{-1}\left(\Delta_{X}\right) .
$$

Furthermore, over a point $\left(\delta, \delta^{\prime}\right) \in F \times F$, the fiber of the map

$$
p^{\prime}:=(p, p)_{\mid \widetilde{I}}: \widetilde{I} \rightarrow F \times F
$$

identifies schematically to the intersection of the corresponding lines $L, L^{\prime}$ in $X$. Thus, away from the diagonal, this fibre is a reduced point, and the restriction $p_{0}^{\prime}$ of $p^{\prime}$ to $\widetilde{I}_{0}:=\widetilde{I} \backslash\left(p^{\prime}\right)^{-1}\left(\Delta_{F}\right)$ is an isomorphism onto $I_{0}$.

Furthermore, as $\widetilde{I}_{0}$ is a local complete intersection, and $(p, p)$ is a submersion, $I_{0}$ is also a local complete intersection, and thus $I_{0}^{2}$ is equal to $j_{*}\left(c_{2}\left(N_{I_{0}}\right)\right)$, where $N_{I_{0}}$ is the normal bundle of $I_{0}$ in $F \times F \backslash \Delta_{F}$ and $j$ is the inclusion of $I_{0}$ in $F \times F \backslash \Delta_{F}$. On the other hand, as $p_{0}^{\prime}$ is an isomorphism onto $I_{0}$, the normal bundle of $\widetilde{I}_{0}$ in $P \times P$ fits into a normal sequence

$$
0 \rightarrow T_{P \times P / F \times F \mid \widetilde{I}_{0}} \rightarrow N_{\widetilde{I}_{0} / P \times P} \rightarrow\left(p_{0}^{\prime}\right)^{*} N_{I_{0} / F \times F} \rightarrow 0 .
$$

We deduce from this that $p_{0}^{\prime *} c_{2}\left(N_{I_{0} / F \times F}\right)$ can be expressed as a polynomial in the Chern classes $c_{1}, c_{2}$ of the normal bundle $N_{\widetilde{I}_{0} / P \times P}$ and in the Chern classes of $T_{P \times P / F \times F \mid \widetilde{I}_{0}}$.

The later ones are polynomials in $h_{1}, l_{1}^{\prime}, h_{2}, l_{2}^{\prime}$, where

$$
h_{i}=p r_{i}^{*} h, l_{i}^{\prime}=p r_{i}^{*}\left(p^{*} l\right), i=1,2,
$$

and $p r_{i}$ are the two projections of $P \times P$ onto $P$. Next we observe that, as $\widetilde{I}=(q, q)^{-1}\left(\Delta_{X}\right)$, we have the equalities

$$
c_{i}\left(N_{\widetilde{I}_{0} / P \times P}\right)=q_{0}^{*} c_{i}\left(T_{X}\right),
$$

where $q_{0}: \widetilde{I}_{0} \rightarrow X$ is the restriction of $(q, q)$ to $\widetilde{I}_{0}$. But $c_{i}\left(T_{X}\right)$ are polynomials in $H$. Thus we conclude that we have a relation:

$$
I_{0}^{2}=p_{0 *}^{\prime}\left(p_{0}^{\prime *} c_{2}\left(N_{I_{0} / F \times F}\right)\right)
$$




$$
=p_{0 *}^{\prime}\left(P\left(h_{i}, l_{i}^{\prime}\right)\right),
$$

for some degree 2 polynomial $P$ in $h_{i}, l_{i \mid \widetilde{I}_{0}}^{\prime}$ (in fact $h_{1}=h_{2}$ on $\widetilde{I}_{0}$ ). This can also be written as

$$
I_{0}^{2}=(p, p)_{*}\left(P\left(h_{i}, l_{i}^{\prime}\right) \cdot \widetilde{I}\right)_{\mid F \times F \backslash \Delta_{F}} .
$$

Let us now write the quadratic polynomial $P$ as

$$
P=h_{1} A+h_{2} B+Q,
$$

where $A, B$ are linear in $h_{i}, l_{i}^{\prime}$, and $Q$ is quadratic in $l_{1}^{\prime}, l_{2}^{\prime}$. We have by the projection formula, noting that $l_{i}^{\prime}=(p, p)^{*} l_{i}$,

$$
(p, p)_{*}\left(Q\left(l_{i}^{\prime}\right) \cdot \widetilde{I}\right)=Q\left(l_{i}\right) \cdot I,
$$

which is of the form $\Gamma^{\prime} \cdot I$.

At this point we proved

$$
I_{0}^{2}=\Gamma^{\prime} \cdot I_{0}+(p, p)_{*}\left(\left(h_{1} A+h_{2} B\right) \cdot \widetilde{I}\right)_{\mid F \times F \backslash \Delta_{F}} .
$$

Finally, we observe that the diagonal of $X$ admits a Künneth type decomposition:

$$
\Delta_{X}=\Delta_{1}+\Delta_{0}
$$

where $\Delta_{1}$ can be written as a sum

$$
\Delta_{1}=\sum_{i} \alpha_{i} H_{1}^{i} \cdot H_{2}^{4-i}
$$

and $\Delta_{0}$ has the property that

$$
H_{1} \cdot \Delta_{0}=0, H_{2} \cdot \Delta_{0}=0 \text { in } C H^{6}(X \times X) .
$$

Here $H_{i}=p r_{i}^{*} H, i=1,2$, and $p r_{i}$ are the two projections on $X \times X$. We obtain this decomposition as follows: we choose the $\alpha_{i}$ in such a way that we have the following equalities between intersection numbers:

$$
\Delta_{X} \cdot H_{1}^{i} \cdot H_{2}^{4-i}=\Delta_{1} \cdot H_{1}^{i} \cdot H_{2}^{4-i}, \text { for } i=0, \ldots, 4 .
$$

Then the cycle $\Delta_{0}=\Delta_{X}-\Delta_{1}$ is such that its image under each inclusion

$$
j_{1}: X \times X \hookrightarrow \mathbb{P}^{5} \times X, j_{2}: X \times X \hookrightarrow X \times \mathbb{P}^{5}
$$

is rationally equivalent to 0 , because $j_{1 *} \Delta_{X}=\Delta_{\mathbb{P}^{5} \mid \mathbb{P}^{5} \times X}$. This implies (3.31) because

$$
j_{1}^{*} \circ j_{1 *}=3 H_{1} \cdot, \quad j_{2}^{*} \circ j_{2 *}=3 H_{2} \cdot
$$


From the decomposition above, and recalling that

$$
\widetilde{I}=(q, q)^{-1}\left(\Delta_{X}\right)=(q, q)^{*} \Delta_{X}, h_{i}=(q, q)^{*} H_{i}
$$

we conclude that

$$
h_{1} A \cdot \widetilde{I}=A \cdot(q, q)^{*}\left(H_{1} \cdot \Delta_{X}\right)=A \cdot(q, q)^{*}\left(H_{1} \Delta_{1}\right) .
$$

But as $H_{1} \Delta_{1}$ is a polynomial in $H_{1}, H_{2}$, it is then clear that $(p, p)_{*}\left(h_{1} A \cdot \widetilde{I}\right)$ is a cycle of the form $\Gamma^{\prime \prime}$ as in the Proposition. Similarly for $(p, p)_{*}\left(h_{2} B \cdot \widetilde{I}\right)$. Thus, using (3.30), the existence of a quadratic relation (3.27) is proven.

We now show that $\alpha \neq 0$. Mimicking the arguments in [22], one sees that there exist an hypersurface $W \subset F$ and a non zero coefficient $\gamma \in \mathbb{Z}$ such that for each $\delta \in F$, there is a relation

$$
\gamma \delta=S_{\delta}^{2}+z,
$$

where $z$ is a 0 -cycle supported on $W$. Here $S_{\delta}$ is the surface of lines of $X$ meeting $\delta$, so that $S_{\delta}=I^{*} \delta$ in $C H^{2}(F)$ and

$$
S_{\delta}^{2}=\gamma \delta-z=\left(I^{2}\right)^{*} \delta \text { in } C H^{4}(F) .
$$

We have an equality

$$
I^{2}=\alpha \Delta_{F}+\Gamma \cdot I+\Gamma^{\prime} \text { in } C H^{4}(F \times F),
$$

from which we deduce that $\left(I^{2}\right)^{*}$ acts as multiplication by $\alpha$ on $H^{4,0}(F) \neq 0$. On the other hand, (3.32) together with the generalized Mumford theorem (cf [23], Proposition 10.24 ), shows that $\left(I^{2}\right)^{*}$ acts as multiplication by $\gamma$ on $H^{4,0}(F)$. Thus $\alpha=\gamma \neq 0$.

We have the following corollary of Proposition 3.3.

Corollary 3.4. Let $z \in C H_{1}(F)=C H^{3}(F)$ be a 1-cycle. Assume that $z$ is rationally equivalent to a combination of rational curves $C_{i} \subset F$,

$$
z=\sum_{i} n_{i} C_{i}
$$

that $z$ is cohomologous to 0 , and that one (or equivalently any) point $x_{i}$ of $C_{i}$ is rationally equivalent to $o$ in $F$. Then $z=0$ in $C H^{3}(F)$. 
Proof. Indeed, observe that since

$$
z=\sum_{i} n_{i} C_{i}
$$

with $C_{i}$ rational, we have

$$
\Delta_{F *} z=\sum_{i} n_{i}\left(x_{i} \times C_{i}+C_{i} \times x_{i}\right) \text { in } C H(F \times F),
$$

where $x_{i}$ is any point of $C_{i}$. Now $I^{2}$ is the restriction of $I \times I$ to the diagonal $\Delta_{F \times F}$ of $F \times F$. Thus we have

$$
\left(I^{2}\right)^{*} z=\left((I \times I)^{*}\left(\Delta_{F *} z\right)\right)_{\mid \Delta_{F}} .
$$

From (3.33), we conclude that

$$
\left(I^{2}\right)^{*} z=2 \sum_{i} n_{i} I^{*} C_{i} \cdot I^{*} x_{i}
$$

By assumption, we have $I^{*} x_{i}=I^{*} o$ in $C H^{2}(F)$, thus (3.34) is equal to

$$
2 I^{*} o \cdot \sum_{i} n_{i} I^{*} C_{i}=2 I^{*} o \cdot I^{*} z .
$$

But $z$ is homologous to 0 , so $I^{*} z \in C H^{1}(F)$ is also homologous to 0 , hence it is rationally equivalent to 0 . Thus $\left(I^{2}\right)^{*} z=0$ in $C H^{3}(F)$.

Now we apply Proposition 3.3 which gives a relation

$$
\alpha z=\left(I^{2}\right)^{*} z-(\Gamma \cdot I)^{*} z-\Gamma^{\prime *} z .
$$

As $\left(I^{2}\right)^{*} z=0$, the right hand side is equal to

$$
-(\Gamma \cdot I)^{*} z-\Gamma^{*} z
$$

But we know that both $I^{*} z$ and $l \cdot z$ are rationally equivalent to 0 : for the first, this was noticed just before, and for the second, this is because it is a multiple of $o$ and homologous to 0 . Hence it follows that $-(\Gamma \cdot I)^{*} z-\Gamma^{*} z=0$ and, as $\alpha \neq 0$, we conclude that $z=0$.

As a consequence, we can start the computation of relations in $C H^{3}(F)$ by showing the following Lemma 3.5: Notice that $\left[l^{3}\right]$ and $[l c]$ are proportional in $H^{6}(F, \mathbb{Q})$. Let this relation be

$$
\left[\mu c l-\nu l^{3}\right]=0 \text { in } H^{6}(F, \mathbb{Q}), \mu \neq 0, \nu \neq 0 .
$$


On the Chow Ring of Certain Algebraic Hyper-Kähler Manifolds 637

Lemma 3.5. We have the equality

$$
\mu c l-\nu l^{3}=0
$$

in $C H^{3}(F)$.

Proof. Indeed, it suffices to prove this relation for generic $X$. In that case, we proved that the cycles $l^{3}$ and $l c$ are supported on a rational surface of class $c$, all points of which are rationally equivalent to $o$ in $F$. Thus the cycle $z=\mu c l-\nu l^{3}$ satisfies the assumptions of Corollary 3.4.

In conclusion, we proved in Lemma 3.2 and Lemma 3.5 that all polynomial cohomological relations between $l$ and $c$ hold in $C H(F)$.

Let us decompose now $C H^{1}(F)$ as

$$
C H^{1}(F)=<l>\oplus C H^{1}(F)_{0},
$$

where $C H^{1}(F)_{0}=p_{*} q^{*} C H^{2}(X)_{\text {prim }}$. Recall the following from [23], 9.3.4. Let $Z \in C H^{2}(X)_{\text {prim }}:=\left\{Z \in C H^{2}(F),[Z] \in H^{4}(X, \mathbb{Q})_{\text {prim }}\right\}$. Write

$$
q^{*} Z=h p^{*} D+p^{*} Z^{\prime}
$$

Then from

$$
H \cdot Z=0 \text { in } C H^{3}(X),
$$

(see [23], 9.3.4), we get, using $h^{2}=h p^{*} l-p^{*} c$,

$$
h^{2} p^{*} D+h p^{*} Z^{\prime}=0=h p^{*}\left(l D+Z^{\prime}\right)-p^{*}(c D) .
$$

Thus we have $D=p_{*} q^{*} Z$, and

$$
Z^{\prime}=-l D, \quad c D=0 \text { in } C H(F) .
$$

In particular

$$
q^{*} Z=\left(h-p^{*} l\right) p^{*} D .
$$

Let us deduce from this the following:

Lemma 3.6. For any $D \in C H^{1}(F)_{0}$, we have the relations:

$$
\begin{aligned}
& l^{2} D^{2}=C q([D]) o, \\
& l D^{2}=C^{\prime} q([D]) E_{x},
\end{aligned}
$$


where $q$ is the Beauville-Bogomolov quadratic form on $H^{2}(F), C, C^{\prime}$ are constants, and $E_{x}=p_{*} q^{*} x$ was already introduced and shown to be proportional to $l^{3}$ and $c l$ in $C H^{3}(F)$.

Proof. Note that since $X$ is Fano, we have $C H^{4}(X)=\mathbb{Q}$ and thus

$$
Z^{2}=<Z, Z>x
$$

for any $x \in X$. Using (3.37), we get

$$
q^{*}\left(Z^{2}\right)=\left(h-p^{*} l\right)^{2} p^{*}\left(D^{2}\right) .
$$

Next we use the relations $c D=0, h^{2}=h p^{*} l-p^{*} c$, and (3.38) to rewrite (3.39) as

$$
\begin{aligned}
<Z, Z>q^{*} x & =h p^{*} l p^{*} D^{2}-2 h p^{*} l p^{*} D^{2}+p^{*}\left(l^{2} D^{2}\right) \\
& =-h p^{*}\left(l D^{2}\right)+p^{*}\left(l^{2} D^{2}\right) .
\end{aligned}
$$

Note now that $\langle Z, Z\rangle=-C^{\prime} q([D])$ for some constant $C^{\prime}$, as proved in [4], so that pushing forward via $p$ the above expression, we get

$$
C^{\prime} q([D]) E_{x}=l D^{2} .
$$

Finally, applying $l$ to this, we get

$$
l^{2} D^{2}=C^{\prime} q(D) l \cdot E_{x}=C q(D) o,
$$

with $C=C^{\prime} \operatorname{deg}\left(l \cdot E_{x}\right)$. (We use (3.26) and Lemma 3.5 to get the last equality.)

Summing-up what we have done up to now, we get:

Proposition 3.7. Any polynomial relation

$$
[P]=0 \text { in } H^{6}(F, \mathbb{Q}) \text { or in } H^{8}(F, \mathbb{Q}),
$$

in the variables $l, c, D \in C H^{1}(F)_{0}$, which is of degree $\leq 2$ in $D$, is already satisfied in $C H^{3}(F)$, resp. $C H^{4}(F)$.

Proof. Indeed, consider first the case of $H^{8}$. The polynomial expression $P$ is then of the form

$$
P=c Q+l^{2} Q^{\prime}+c l A+l^{3} A^{\prime}+\alpha c^{2}+\beta c l^{2}+\gamma l^{4},
$$


where $Q, Q^{\prime} \in S^{2} C H^{1}(F)_{0}, A, A^{\prime} \in C H^{1}(F)_{0}$ and $\alpha, \beta, \gamma$ are constants. But we know (cf (3.36)) that

$$
c Q=0, c A=0,
$$

and that $l^{2} Q^{\prime}, c^{2}, c l^{2}, \gamma l^{4}$ are all multiples of $o$ (cf Lemma 3.2, Lemma 3.6). On the other hand, as we proved that the cycle $l^{3}$ is rationally equivalent to a cycle supported on a rational surface in the class $c$, and all points of $\Sigma$ are rationally equivalent to $o$, it follows that $l^{3} A^{\prime}$ is also a multiple of $o$. Thus $P$ is a multiple of $o$ in $C H^{4}(F)$, and as it is cohomologous to 0 , it must be 0 .

Next we consider the case of degree 6 . Then $P$ can be written as

$$
P=l Q+c A+l^{2} A^{\prime}+\alpha l^{3}+\beta c l,
$$

where $Q \in S^{2} C H^{1}(F)_{0}, A, A^{\prime} \in C H^{1}(F)_{0}$ and $\alpha, \beta$ are constants.

We know that $c A=0$ and we proved already that the cycles

$$
l Q, l^{3}, c l
$$

are all proportional in $C H^{3}(F)$ (cf Lemma 3.5, Lemma 3.6). Using these proportionality relations, we get an equality in $C H(F)$ :

$$
P=l^{2}\left(A^{\prime}+\gamma l\right),
$$

where the number $\gamma$ depends on $Q, \alpha, \beta$ and involves the constants $\mu, \nu, C^{\prime}$ of Lemma 3.5, Lemma 3.6. But we know that $[P]=0$, and thus the hard Lefschetz theorem implies that $\left[A^{\prime}+\gamma l\right]=0$. Thus, as we are in $C H^{1}(F) \subset H^{2}(F, \mathbb{Q})$, we have $A^{\prime}+\gamma l=0$ and $P=0$.

We now turn to polynomials of degree at most 3 in $D$. Let us first consider the case of polynomials of degree 4 , that is $P \in C H^{4}(F)$.

Lemma 3.8. Any polynomial expression $P \in C H^{4}(F)$ in $l, c, D \in C H^{1}(F)_{0}$ which is of degree at most 3 in $D$ is a multiple of o. Thus, if $[P]=0$ in $H^{8}(F, \mathbb{Q})$, then $P=0$.

Proof. Indeed this was already proved for polynomial expressions of degree at most 2 in $D$ (cf Proposition 3.7), and thus, we only have to consider expressions of the form

$$
P=l T
$$


where $T \in S^{3} C H^{1}(F)_{0}$. Now Lemma 3.6 says that for $D \in C H^{1}(F)_{0}, l D^{2}$ is proportional to $l^{3}$ in $C H^{3}(F)$. Hence $l D^{3}$ is proportional to $l^{3} D$ in $C H^{4}(F)$. But by Proposition 3.7, we know that $l^{3} D$ is a multiple of $o$ in $C H^{4}(F)$, as is any polynomial expression of degree $\leq 2$ in $D$.

We turn now to the cubic polynomial relations in $C H^{3}(F)$. First of all we have the following lemma:

Lemma 3.9. For any $D \in C H^{1}(F)_{0}$, one has

$$
\left[D^{3}\right]=\frac{3}{q([l])} q([D])\left[l^{2} D\right] .
$$

Proof. Recall from [18], [9] that, in the complex cohomology algebra $H^{*}(F, \mathbb{C})$, one has the relations

$$
d^{\prime 3}=0
$$

for $d^{\prime} \in H^{2}(F, \mathbb{C})$ such that $q\left(d^{\prime}\right)=0$.

It follows that we have more generally a relation of the form

$$
d^{\prime 3}=q\left(d^{\prime}\right) A\left(d^{\prime}\right)
$$

where $A\left(d^{\prime}\right) \in H^{6}(F, \mathbb{C})$ is a linear function of $d^{\prime}$. We apply this to $d^{\prime}=d+\lambda[l]$, where $\lambda \in \mathbb{C}, d=[D], D \in C H^{1}(F)_{0}$. Then we get, recalling that $q(d,[l])=0$,

$(3.41) d^{3}+3 \lambda d^{2}[l]+3 \lambda^{2} d[l]^{2}+\lambda^{3}[l]^{3}=\left(q(d)+\lambda^{2} q([l])\right) A\left(d^{\prime}\right)$ in $H^{6}(F, \mathbb{C})$.

Write $A\left(d^{\prime}\right)=a(d) N+\lambda M$. Then we get by taking the 0 -th order term in $\lambda$ :

$$
d^{3}=q(d) a(d) N .
$$

The order 2 term in $\lambda$ gives now

$$
3 d[l]^{2}=q([l]) a(d) N,
$$

from which we conclude that

$$
d^{3}=\frac{3}{q([l])} q(d) l^{2} d
$$

We will show the following proposition. 
Proposition 3.10. For any $D \in C H^{1}(F)_{0}$, we have the relation

$$
D^{3}=\frac{3}{q([l])} q([D]) l^{2} D \text { in } C H^{3}(F) .
$$

Postponing the proof of Proposition 3.10, we conclude now the proof of Theorem 3.1 , or equivalently of Theorem $1.4,2$ ).

Proof of Theorem 3.1. Let us first treat the case of a polynomial expression $P \in C H^{3}(F)$, which has to be of degree at most 3 in $P i c F_{0}$. So assume $[P]=0$, where $P=T+l Q+l^{2} L+c L^{\prime}+C$, is the decomposition of $P$ into elements of $\operatorname{Sym}^{C} \mathrm{CH}^{1}(F)_{0}$ of degree 3,2, 1 and 0 respectively, whose coefficients are polynomials in $c, l$. We know from (3.36) that $c L^{\prime}=0$. We also know from Lemma 3.6 and Lemma 3.5 that $l Q$ and $C$ are proportional to $l^{3}$ in $C H^{3}(F)$. Thus we have

$$
l Q+C=\gamma l^{3} \text { in } C H^{3}(F) .
$$

Finally, it follows from Proposition 3.10 that $T$ is equal in $C H^{3}(F)$ to $l^{2} D$ for some $D \in$ Pic $F_{0}$.

Thus we have $P=l^{2}(D+L)+\gamma l^{3}$ in $C H^{3}(F)$ and the relation $[P]=0$ implies

$$
\left[l^{2}\right][D+L+\gamma l]=0 \text { in } H^{6}(F, \mathbb{Q}) .
$$

But the hard Lefschetz theorem implies then that $[D+L+\gamma l]=0$. Thus $D+L+\gamma l=0$ and $P=0$.

To conclude the proof of the theorem, we now have to consider the case of a polynomial $P \in C H^{4}(F)$ of degree 4 in $D \in C H^{1}(F)_{0}$. But Proposition 3.10 shows that, for any $D \in C H^{1}(F)_{0}$, we have the relation

$$
D^{4}=\frac{3}{q([l])} q([D]) l^{2} D^{2} \text { in } C H^{4}(F) .
$$

We proved in Lemma 3.6 that $l^{2} D^{2}$ is proportional to $o$ in $C H^{4}(F)$. Thus $D^{4}$ is a multiple of $o$ and so is any quartic homogeneous polynomial expression in $D \in$ Pic $F_{0}$.

By Lemma 3.8, the same is true of any polynomial expression of degree $\leq$ 3 in $D$, with coefficients which are polynomials in $l, c$. Thus any polynomial expression $P$ of degree 4 in $D$, with coefficients in $l, c$ is a multiple of $o$ in $C H^{4}(F)$. In particular, if $[P]=0$, we have $P=0$. 
Proof of Proposition 3.10. We first prove the result under the assumption that $X$ contains no plane. We will show later on how to deduce the result when $X$ contains planes.

Let us introduce the following object:

$$
\widetilde{F}=\left\{\left(\delta_{1}, \delta_{2}\right) \in F \times F, \exists P \cong \mathbb{P}^{2} \subset \mathbb{P}^{5}, P \cap X=2 \delta_{1}+\delta_{2}\right\} .
$$

Because we made the assumption that $X$ does not contain any plane, $\widetilde{F}$ is irreducible, and is the graph of the rational map $\phi: F \rightarrow F$ described in [21]. We shall denote by

$$
\tau: \widetilde{F} \rightarrow F, \tilde{\phi}: \widetilde{F} \rightarrow F
$$

the restrictions to $\widetilde{F}$ of the two projections. Thus $\tau$ is birational and $\phi=\tilde{\phi} \circ \tau^{-1}$.

Note that $\widetilde{F}$ may be singular, which may imply that the groups $C H_{i}(\widetilde{F})$ and $C H^{4-i}(\widetilde{F})$ differ, and cause troubles because on one hand we compute relations in $C H_{*}(\widetilde{F})$, and on the other hand, we use intersection product on $C H(\widetilde{F})$. However, there is a desingularization of $\widetilde{F}$ which is obtained by a sequence of blowups starting from $F$. We leave to the reader to adapt the following arguments using this smooth model, and in the sequel, we do as if $\widetilde{F}$ were smooth.

We will prove the following two Lemmas:

Lemma 3.11. For $D \in C H^{1}(F)_{0}$, we have $\tilde{\phi}^{*} D=-2 \tau^{*} D$ in $C H^{1}(\widetilde{F})$.

Lemma 3.12. Let $I \subset F \times F$ be the incidence subscheme defined in 3.24. Then

$$
(\tilde{\phi}, I d)^{*} I=-2(\tau, I d)^{*} I+Z
$$

in $C H^{2}(\widetilde{F} \times F)$, where $Z$ is a cycle of the form

$$
Z=Z_{1} \times F+D^{\prime} \times l+\widetilde{F} \times Z_{2},
$$

with $Z_{1} \subset \widetilde{F}$ a codimension 2 cycle, $D^{\prime} \subset \widetilde{F}$ a codimension 1 cycle, $Z_{2} \subset F a$ codimension 2 cycle.

Assuming these lemmas, let us show how to conclude the proof: First of all, from Lemma 3.11, we deduce that for $D \in P i c F_{0}$, we have

$$
\tilde{\phi}^{*} D^{3}=-8 \tau^{*} D^{3} \text { in } C H^{3}(\widetilde{F}) .
$$

Next, from lemma 3.12, we deduce that

$$
(\tilde{\phi}, I d)^{*} I^{2}=4(\tau, I d)^{*} I^{2}-4 Z \cdot(\tau, I d)^{*} I+Z^{2} .
$$


Note now that by definition of $\phi^{*}$ :

$$
\tau_{*} \circ \tilde{\phi}^{*}=\phi^{*},
$$

acting on $C H(F)$. Furthermore we have, applying $\tau_{*},(\tau, I d)_{*}$ to $(3.45),(3.46)$ :

$$
\begin{array}{r}
\phi^{*} D^{3}=-8 D^{3}, \\
(\phi, I d)^{*} I^{2}=4 I^{2}-4 I \cdot Z^{\prime}+Z^{\prime \prime},
\end{array}
$$

where $Z$ is defined in (3.43) and

$$
Z^{\prime}:=(\tau, I d)_{*} Z, Z^{\prime \prime}=(\tau, I d)_{*} Z^{2} .
$$

Observe now that

$$
\phi^{*}\left(\left(I^{2}\right)^{*}(z)\right)=\left((\phi, I d)^{*}\left(I^{2}\right)\right)^{*}(z), \forall z \in C H_{1}(F) .
$$

Combining this with (3.48) and the quadratic relation (3.27) given in Proposition 3.3 , we get, for any $z \in C H_{1}(F)$ :

$$
\begin{array}{r}
\phi^{*}\left(\alpha z+(\Gamma \cdot I)^{*} z+\left(\Gamma^{\prime}\right)^{*} z\right)=4\left(I^{2}\right)^{*} z-4\left(I \cdot Z^{\prime}\right)^{*} z+\left(Z^{\prime \prime}\right)^{*} z \\
=4\left(\alpha z+(\Gamma \cdot I)^{*} z+\left(\Gamma^{\prime}\right)^{*} z\right)-4\left(I \cdot Z^{\prime}\right)^{*} z+\left(Z^{\prime \prime}\right)^{*} z .
\end{array}
$$

Applying this to $z=D^{3}$ and using (3.47), we finally get

$$
\begin{array}{r}
-8 \alpha D^{3}+\phi^{*}\left((\Gamma \cdot I)^{*} D^{3}+\left(\Gamma^{\prime}\right)^{*} D^{3}\right) \\
=4 \alpha D^{3}+4\left((\Gamma \cdot I)^{*} D^{3}+\left(\Gamma^{\prime}\right)^{*} D^{3}-\left(I \cdot Z^{\prime}\right)^{*} D^{3}\right)+\left(Z^{\prime \prime}\right)^{*} D^{3} .
\end{array}
$$

In conclusion, we proved that

$\left.12 \alpha D^{3}=\phi^{*}\left((\Gamma \cdot I)^{*} D^{3}+\left(\Gamma^{\prime}\right)^{*} D^{3}\right)-4\left((\Gamma \cdot I)^{*} D^{3}+\left(\Gamma^{\prime}\right)^{*} D^{3}\right)-\left(I \cdot Z^{\prime}\right)^{*} D^{3}\right)-\left(Z^{\prime \prime}\right)^{*} D^{3}$.

We claim now that $\left(\Gamma^{\prime}\right)^{*} D^{3}, \phi^{*}\left(\left(\Gamma^{\prime}\right)^{*} D^{3}\right)$ and $\left(Z^{\prime \prime}\right)^{*} D^{3}$ are all multiples of $l^{3}$ (or equivalently $c l$ ).

In the case of $\left(\Gamma^{\prime}\right)^{*} D^{3}$, this is a consequence of the fact that $\Gamma^{\prime} \in C H^{4}(F \times F)$ is a polynomial in $p r_{1}^{*} l, p r_{2}^{*} l, p r_{1}^{*} c, p r_{2}^{*} c$, and of lemma 3.5. This implies also the claim for $\phi^{*}\left(\left(\Gamma^{\prime}\right)^{*} D^{3}\right)$, as one shows easily (using Lemma 3.5) that $\phi^{*} l^{3}$ is a multiple of $l^{3}$. As for $\left(Z^{\prime \prime}\right)^{*} D^{3}$, we observe that we have for any $z \in C H_{1}(F)$,

$$
\left(Z^{\prime \prime}\right)^{*} z=\tau_{*}\left(\left(Z^{2}\right)^{*} z\right),
$$

and using formula (3.44) for $Z$, this gives

$$
\left(Z^{\prime \prime}\right)^{*} z=2 \tau_{*}\left(Z_{1} D^{\prime}\right) \operatorname{deg}(l \cdot z) .
$$


Hence it suffices to show that $\tau_{*}\left(Z_{1} D^{\prime}\right)$ is a multiple of $l^{3}$. Now we have by (3.51) applied to $l^{3}$ :

$$
\left(Z^{\prime \prime}\right)^{*} l^{3}=2 \tau_{*}\left(Z_{1} D^{\prime}\right) \operatorname{deg} l^{4} .
$$

Thus it suffices to show that $\left(Z^{\prime \prime}\right)^{*} l^{3}$ is a multiple of $l^{3}$. This follows now from (3.49) applied to $z=l^{3}$, and from the fact that

$$
\phi^{*} l^{3},(\Gamma \cdot I)^{*} l^{3},\left(\Gamma^{\prime}\right)^{*} l^{3},\left(I \cdot Z^{\prime}\right)^{*} l^{3}
$$

are all multiples of $l^{3}$. For the first three, this follows easily from the definition of $\phi$ and from the form of $\Gamma, \Gamma^{\prime}$; for the last one, this follows from the fact that, for any $z \in C H_{1}(F),\left(I \cdot Z^{\prime}\right)^{*} z$ is a linear combination of $\tau_{*}\left(Z_{1}\right) \cdot I^{*}(z)$ and $\tau_{*} D^{\prime} \cdot I^{*}(l z)$. Then the result is a consequence of the fact that

$$
I^{*} l^{3}, I^{*} l^{4}, \tau_{*}\left(Z_{1}\right), \tau_{*} D^{\prime}
$$

are polynomial expressions in $l$ and $c$, which is proved using (3.43) and the definitions of $\widetilde{F}$ and $I$.

Next recall that the codimension 2-cycle $\Gamma$ is a linear combination of $l_{1}^{2}, l_{2}^{2}, l_{1} l_{2}$ on $F \times F$. Thus $(\Gamma \cdot I)^{*} D^{3}$ is a combination of $l^{2} I^{*}\left(D^{3}\right)$ and of $l I^{*}\left(l D^{3}\right)$. Next, for the same reason, $\left(I \cdot Z^{\prime}\right)^{*} D^{3}$ is a linear combination of $\tau_{*}\left(Z_{1}\right) \cdot I^{*}\left(D^{3}\right)$ and $\tau_{*} D^{\prime} \cdot I^{*}\left(l D^{3}\right)$, that is of

$$
l^{2} \cdot I^{*}\left(D^{3}\right), c \cdot I^{*}\left(D^{3}\right), l \cdot I^{*}\left(l D^{3}\right) .
$$

Thus our relation (3.50) becomes:

$$
\begin{aligned}
12 \alpha D^{3}=\phi^{*}\left(\mu l^{2} I^{*}\left(D^{3}\right)\right. & \left.+\nu l I^{*}\left(l D^{3}\right)\right) \\
& +\mu^{\prime} l^{2} I^{*}\left(D^{3}\right)+\nu^{\prime} l I^{*}\left(l D^{3}\right)+\mu^{\prime \prime} c I^{*}\left(D^{3}\right)+\mu^{\prime \prime \prime} l^{3} .
\end{aligned}
$$

Recall from Lemma 3.8 that $l D^{3}$ is proportional to $o$. Thus $l I^{*}\left(l D^{3}\right)$ is proportional to $l I_{o}$ which is a multiple of $l^{3}$ and $c l$ in $C H^{3}(F)$. Furthermore, we mentioned already that $\phi^{*}\left(l^{3}\right)$ is also proportional to $l^{3}$.

Next we have

Lemma 3.13. For some constant $\beta$, and for any $D \in C H^{1}(F)_{0}$, one has

$$
I^{*}\left(D^{3}\right)=\beta q([D]) D .
$$


Proof. Indeed, as we are in $C H^{1}(F)$, it suffices to show this in $H^{2}(F, \mathbb{Q})$. But we know that $\left[D^{3}\right]=\frac{3}{q(l)} q(D)\left[l^{2} D\right]$. Thus it suffices to show that for some constant $\beta^{\prime}$, and for any $[D] \in H^{2}(F, \mathbb{Q})_{0}$,

$$
\left.I^{*}\left([l]^{2}[D]\right)\right]=\beta^{\prime}[D] .
$$

This is immediate because the left hand side is a morphism of Hodge structure from $H^{2}(F, \mathbb{Q})_{0}$ to $H^{2}(F, \mathbb{Q})$ which is defined for general $X$, hence has to be a multiple of the identity, because the Hodge structure on $H^{2}(F, \mathbb{Q})_{0}$ for general $X$ is simple with $h^{2,0}=1$, while $H^{2}(F, \mathbb{Q})=H^{2}(F, \mathbb{Q})_{0}+\mathbb{Q}[l]$.

From this lemma, we get in particular that $c I^{*}\left(D^{3}\right)=0$, and we deduce from (3.52) a relation:

$$
12 \alpha D^{3}=\phi^{*}\left(\mu q([D]) l^{2} D\right)+\mu_{1}^{\prime} q([D]) l^{2} D+\nu^{\prime} l^{3} .
$$

Furthermore, we recall that by Lemma 3.11

$$
\tilde{\phi}^{*} D=-2 \tau^{*} D \text {. }
$$

Hence it follows that

$$
\phi^{*}\left(l^{2} D\right)=\tau_{*}\left(-2 \tau^{*} D \tilde{\phi}^{*} l^{2}\right)=-2 D \phi^{*} l^{2} .
$$

It is easy to verify that $\phi^{*} l^{2}$ is a combination of $l^{2}$ and $c$. As $c D=0$, we conclude that $\phi^{*}\left(l^{2} D\right)$ is a multiple of $l^{2} D$. Thus we finally proved that we have a relation

$$
12 \alpha D^{3}=\mu^{\prime \prime} q([D]) l^{2} D+\nu^{\prime} l^{3} .
$$

On the other hand, we know that we have the cohomological relation

$$
\left[D^{3}\right]=\frac{3}{q(l)} q([D])\left[l^{2} D\right] .
$$

Using the hard Lefschetz theorem, and comparing with the cohomological relation

$$
12 \alpha\left[D^{3}\right]=\mu^{\prime \prime} q([D])\left[l^{2} D\right]+\nu^{\prime \prime}\left[l^{3}\right]
$$

deduced from (3.53), we conclude that $\nu^{\prime}=0$, and that

$$
\frac{\mu^{\prime \prime}}{12 \alpha}=\frac{3}{q(l)} \text {. }
$$

This concludes the proof of Proposition 3.10 when $X$ contains no plane.

It remains to see how to do when $X$ contains a plane. Let $D:=D_{Z}$ for some primitive class $[Z] \in H^{4}(X, \mathbb{Q})$. In that case, either $[Z]$ is a multiple of the primitive component $[H]^{2}-3[\mathbb{P}]$ of the cohomology class of a plane $\mathbb{P} \subset X$, or 
it is not. In the later case, one can show by deformation theory that a generic deformation of $X$ preserving the class $Z$ does not preserve any plane contained in $X$. Then we know that (3.42) is satisfied by $D_{t} \in P i c F_{t}$ for the generic member of a family of deformations of the pair $(D, F)$. Thus it is also satisfied by $(D, F)$.

Thus it remains only to consider the case where $D=D_{Z},[Z]=[H]^{2}-3[\mathbb{P}]$. Thus $D=l-3 D_{\mathbb{P}}$, where $D_{\mathbb{P}}$ is the divisor of lines meeting $P$. But this case is easy because away from the dual plane $\mathbb{P}^{*} \subset F, D_{\mathbb{P}}$ is isomorphic via $p$ to $\widetilde{D}:=q^{-1}(\mathbb{P}) \subset P$. It follows that the restriction $\left(D_{\mathbb{P}}\right)_{\mid D_{\mathbb{P}}}$ identifies away from $\mathbb{P}^{*}$ as $\operatorname{det} q^{*} N_{\mathbb{P} / X}-T_{P / F \mid \widetilde{D}}$, that is to the restriction of a combination of $h$ and $l$ to $\widetilde{D}$. From this, one deduces easily that $(3.42)$ is satisfied in $F \backslash \mathbb{P}^{*}$, and as it is satisfied in cohomology, while

$$
C H_{1}\left(\mathbb{P}^{*}\right)=H_{2}\left(\mathbb{P}^{*}, \mathbb{Z}\right)=\mathbb{Z} \subset H^{3}(F, \mathbb{Q}),
$$

it follows that it is satisfied as well on $F$.

Thus Proposition 3.10 is proved, modulo Lemmas 3.11 and 3.12 .

Proof of Lemma 3.11. Note that $\tau: \widetilde{F} \rightarrow F$ is the contraction of a ruled divisor $E$ to the surface $T$ of points $l \in F$ having the property that there is a $\mathbb{P}_{l}^{3} \subset \mathbb{P}^{5}$ which is everywhere tangent to the corresponding line $\Delta_{l} \subset X$. (One verifies that $T$ is always a surface, and the fiber of $\tau$ over $l \in T$ identifies to the $\mathbb{P}^{1}$ parameterizing planes $\mathbb{P}^{2}$ contained in $\mathbb{P}_{l}^{3}$ and containing $\Delta_{l}$, because $X$ contains no plane.)

Thus for any divisor $D \in C H^{1}(F)$, there must be a relation

$$
\tilde{\phi}^{*} D=\tau^{*} D^{\prime}+\sum_{i} \alpha_{i} E_{i} \text { in } C H_{3}(\widetilde{F}),
$$

where the $E_{i}$ are the irreducible components of $E$. Here the $\alpha_{i}$ are computed as $D \cdot \tilde{\phi}\left(E_{i, l}\right)$, where $E_{i, l}$ is the fiber of $E_{i}$ over $l \in T_{i}$. (Here $T_{i}$ is the irreducible component of $T$ corresponding to $E_{i}$.) However, the curve $\tilde{\phi}\left(E_{i, l}\right)$ is the family of lines contained in a cubic surface $S$ in $X$ which is singular along the line $\Delta_{l}$. Thus the surface in $X$ swept out by the lines parameterized by $\tilde{\phi}\left(E_{i, l}\right)$ is the cubic surface $S$, and for $D=D_{Z}$, with $Z \subset X$ a cycle with primitive cohomology class, one has

$$
\alpha_{i}=-D \cdot \tilde{\phi}\left(E_{i, l}\right)=<Z, S>=0
$$


Thus we have

$$
\tilde{\phi}^{*} D=\tau^{*} D^{\prime} \text { in } C H_{3}(\widetilde{F}),
$$

and clearly $D^{\prime}=\phi^{*} D \in C H^{1}(F)$. But the action of $\phi^{*}$ on $C H^{1}(F)_{0}$ is the restriction of the action of $\phi^{*}$ on $H^{2}(F, \mathbb{Q})_{0}:=p_{*} q^{*} H^{4}(X, \mathbb{Q})_{\text {prim }}$. This action is multiplication by -2 , because it is multiplication by -2 on $H^{2,0}(F)$ (cf [21]), and for general $X$ the Hodge structure on $H^{2}(F, \mathbb{Q})_{0}$ is simple. Thus $D^{\prime}=-2 D$ and the lemma is proven.

Proof of Lemma 3.12. We observe first that it suffices to prove the lemma for generic $F$, because the family of $\tilde{F}$ parameterized by the set $U \subset \mathbb{P}\left(H^{0}\left(\mathcal{O}_{\mathbb{P}^{5}}(3)\right)\right)$ corresponding to smooth cubic hypersurfaces which do not contain a plane is flat.

Next we note that because $P i c^{0} F=0$, (which implies that divisors on any product $K \times F$ are rationally equivalent to sum of pull-backs of divisors on each factor, ), and Pic $F=\mathbb{Z} l$, which implies that divisors on $F$ are rationally equivalent to a multiple of $l$, any codimension 2 cycle in $\widetilde{F} \times F$ which is supported on $D \times F$ is of the form

$$
Z_{1} \times F+D^{\prime} \times l,
$$

where $Z_{1}, D^{\prime}$ have respectively codimension 2 and 1 in $\widetilde{F}$.

We use now the fact that for $L \in F$, the points $L$ and $\phi(L)$ of $F$ parameterize lines

$$
\Delta_{L}, \Delta_{\phi(L)}
$$

in $X$ which satisfy the property

$$
2 \Delta_{L}+\Delta_{\phi(L)}=H^{3} \text { in } C H^{3}(X) .
$$

Thus we also have

$$
2 I_{L}+I_{\phi(L)}=C \text { in } C H^{2}(F),
$$

where $C=p_{*} q^{*} H^{3}$ is a constant. We then apply the Bloch-Srinivas argument [6] $([23], 10.3 .1)$, to conclude that $2(\tau, I d)^{*} I+(\tilde{\phi}, I d)^{*} I$ is rationally equivalent to the sum of a cycle of the form $\widetilde{F} \times C$ and of a cycle $W$ supported (via the first projection) on a divisor of $\widetilde{F}$. We can thus apply the remark above, which gives

$$
2(\tau, I d)^{*} I+(\tilde{\phi}, I d)^{*} I=\widetilde{F} \times C+Z_{1} \times F+D^{\prime} \times l,
$$

that is formula (3.44) with $Z_{2}=C$. 


\section{REFERENCES}

[1] D. Abramovich, T. Graber, A. Vistoli. Algebraic orbifold quantum products. Orbifolds in mathematics and physics (Madison, WI, 2001), 1-24, Contemp. Math., 310, Amer. Math. Soc., Providence, RI, 2002.

[2] A. Beauville. Variétés kähleriennes dont la première classe de Chern est nulle, J. Differential Geometry 18 (1983), 755-782.

[3] A. Beauville. On the splitting of the Bloch-Beilinson filtration, in Algebraic cycles and motives (vol. 2), London Math. Soc. Lecture Notes 344, 38-53; Cambridge University Press (2007).

[4] A. Beauville, R. Donagi. La variété des droites d'une hypersurface cubique de dimension 4, C.R. Acad. Sc. Paris 301, 703-706 (1985).

[5] A. Beauville, C. Voisin. On the Chow ring of a K3 surface, J. Algebraic Geom. 13 (2004), no. 3, 417-426.

[6] S. Bloch, V. Srinivas. Remarks on correspondences and algebraic cycles, Amer. J. of Math. 105 (1983) 1235-1253.

[7] F. A. Bogomolov. The decomposition of Kähler manifolds with a trivial canonical class. (Russian) Mat. Sb. (N.S.) 93(135) (1974), 573-575

[8] F. A. Bogomolov. Hamiltonian Kählerian manifolds. (Russian) Dokl. Akad. Nauk SSSR 243 (1978), no. 5, 1101-1104.

[9] F. A. Bogomolov. On the cohomology ring of a simple hyper-Kähler manifold (on the results of Verbitsky). Geom. Funct. Anal. 6 (1996), no. 4, 612-618.

[10] T. Bridgeland, A. King, M. Reid. Mukai implies McKay: the McKay correspondence as an equivalence of derived categories, J. Amer. Math. Soc. 14 (2001), no. 3, 535-554.

[11] M.A. de Cataldo, L. Migliorini: The Chow groups and the motive of the Hilbert scheme of points on a surface, Journal of algebra 251 (2002), 824-848.

[12] H. Clemens, Ph. Griffiths. The intermediate jacobian of the cubic threefold, Ann. of Math. (2) 95 (1972), 281-356.

[13] G. Ellingsrud, L. Göttsche, M. Lehn. On the cobordism class of the Hilbert scheme of a surface. Journal of Algebraic Geometry, 10 (2001), 81 - 100.

[14] B. Fantechi, L. Göttsche. Orbifold cohomology for global quotients, Duke Math J., vol. 117 (2003), 197-227.

[15] M. Haiman. Hilbert schemes, polygraphs, and the Macdonald positivity conjecture, J. Amer. Math. Soc. 14 (2001), 941-1006.

[16] M. Lehn, C. Sorger. The Cup Product of the Hilbert Scheme for K3 Surfaces. Inventiones mathematicae 152 (2003) 305 - 329.

[17] L. Scala. Ph. D. Thesis (2005).

[18] M. Verbitsky. Cohomology of compact hyper-Kähler manifolds and its applications. Geom. Funct. Anal. 6 (1996), no. 4, 601-611.

[19] B. Toen. Théorèmes de Riemann-Roch pour les champs de Deligne-Mumford. (French) K-Theory 18 (1999), no. 1, 33-76. 
[20] G. Vezzosi, A. Vistoli. Higher algebraic $K$-theory of group actions with finite stabilizers. Duke Math. J. 113 (2002), no. 1, 1-55.

[21] C. Voisin. Intrinsic pseudo-volume forms and $K$-correspondences, The Fano Conference, 761-792, Univ. Torino, Turin, 2004.

[22] C. Voisin. Relations dans l'anneau de Chow de la surface des coniques des variétés de Fano, Amer. J. of Mathematics 112 (1990) 877-898.

[23] C. Voisin. Hodge Theory and Complex Algebraic Geometry II, Cambridge studies in advanced mathematics 77, Cambridge Univ. Press (2003).

Claire Voisin

Institut de mathématiques de Jussieu, CNRS,UMR 7586

E-mail: voisin@math.jussieu.fr 